\title{
Multipole interaction between atoms and their photonic environment
}

\author{
Martijn Wubs, ${ }^{1,2, *}$ L. G. Suttorp, ${ }^{3}$ and A. Lagendijk ${ }^{1}$ \\ ${ }^{1}$ Complex Photonic Systems, Faculty of Science and Technology, University of Twente, P.O. Box 217, NL-7500 AE Enschede, \\ The Netherlands \\ ${ }^{2}$ Van der Waals-Zeeman Institute, University of Amsterdam, Valckenierstraat 65, NL-1018 XE Amsterdam, The Netherlands \\ ${ }^{3}$ Institute for Theoretical Physics, University of Amsterdam, Valckenierstraat 65, NL-1018 XE Amsterdam, The Netherlands
}

(Received 1 April 2003; published 28 July 2003)

\begin{abstract}
Macroscopic field quantization is presented for a nondispersive photonic dielectric environment, both in the absence and presence of guest atoms. Starting with a minimal-coupling Lagrangian, a careful look at functional derivatives shows how to obtain Maxwell's equations before and after choosing a suitable gauge. A Hamiltonian is derived with a multipolar interaction between the guest atoms and the electromagnetic field. Canonical variables and fields are determined and in particular, the field canonically conjugate to the vector potential is identified by functional differentiation as minus the full displacement field. An important result is that inside the dielectric a dipole couples to a field that is neither the (transverse) electric nor the macroscopic displacement field. The dielectric function is different from the bulk dielectric function at the position of the dipole, so that local-field effects must be taken into account.
\end{abstract}

DOI: 10.1103/PhysRevA.68.013822

PACS number(s): 42.50.Ct, 42.65.Pc, 41.20.Jb

\section{INTRODUCTION}

Optical properties of atoms, such as spontaneousemission rates, can be strongly influenced by their dielectric environment [1]. It is well known that near a mirror, emission rates can be enhanced or diminished, depending on the distance to the mirror and the orientation of the atomic dipole moment [2,3]. Inside optical cavities, lifetime effects are even stronger [4]. In three-dimensional photonic crystals with a photonic band gap, spontaneous emission would even be fully inhibited at any position in the crystal for atomic transition frequencies within the band gap [5]. Not only single-atom properties but also properties of two or several of atoms, such as dipole-dipole interactions and superradiance, will be influenced by the dielectric environment. Again, extreme changes compared to free space can occur for atoms positioned inside microcavities [6,7] or photonic crystals $[8,9]$.

The above medium-modified processes must be described by a quantum optical theory of dielectrics. In this paper such a theory will be given of guest atoms interacting with a photonic dielectric environment that is characterized by a given spatially varying and real dielectric function $\varepsilon(\mathbf{r})$. The guest atoms, by definition, are the atoms which are not included in the dielectric function. These guests will be described microscopically, whereas the dielectric is described macroscopically in terms of the dielectric function only.

Dielectric mirrors and photonic crystals are usually described by a frequency-independent spatially varying refractive index. Optical components such as glass plates, lenses, and optical cavities are some more examples. In many cases, the refractive index can be considered as piecewise constant but not always; in the so-called graded-index optical fibres the refractive index in the core varies parabolically with the

\footnotetext{
*Electronic address: c.m.wubs@tn.utwente.nl; URL: http:// tnweb.tn.utwente.nl/cops/
}

radius [10]. In this paper, the relative dielectric function $\varepsilon(\mathbf{r})$ is left unspecified (but assume it to be piecewise continuously differentiable) so that the theory describes both piecewise constant and continuously varying dielectric functions.

The quantization of the electromagnetic field in free space can be found in many textbooks on quantum optics [11-14]. In Refs. [15-18], the more general problem is addressed how to quantize the electromagnetic field in a dielectric described by a given real dielectric function $\varepsilon(\mathbf{r})$ that depends on position. The term "macroscopic quantization" has been coined for this procedure [17]. The special case of infinite photonic crystals is treated in Ref. [19].

Guest atoms can be described theoretically essentially in two ways. The simplest way is to treat them as known probes of the electromagnetic field in the medium. In that case, the electromagnetic fields are found from Maxwell's equations in the absence of the guest atoms; the guests are introduced as atoms with given properties such as transition frequencies and dipole moments. The atoms are assumed to couple to the fields that were found in their absence. The second, and more fundamental, way to introduce guest atoms into the theory is to start with Maxwell's equations that also contain as sources the charges that make up the guest atoms. In this second approach, a Hamiltonian for the combined system of charges and fields should be found, which leads to Maxwell's equations, both inside and outside the atoms.

Even for an atom in free space, the difference between these two approaches has led to debates on how to interpret the field to which a dipole couples, either to the transverse part of the electric field or to the displacement field [20-24]. The latter coupling is the correct (more fundamental) interpretation [13] but for most observables, there are no numerical punishments when interpreting the field wrongly. However, inside a dielectric, there would be a considerable difference between an atom coupling to the electric field or to the displacement field. In this paper, it will be shown by using the second, more fundamental, way of introducing guest atoms that neither dipole coupling is correct in a di- 
electric. Moreover, the need to consider local-field effects will arise in a natural way.

Of course, one does not tell the whole truth about a dielectric when describing it by a real, nondispersive, and spatially varying dielectric function $\varepsilon(\mathbf{r})$. In a macroscopic description one forgets details of the microscopic constituents of the dielectric. Also, material dispersion and absorption of light (transitions to nonradiative states in the dielectrics) are neglected. It is well known that the dielectric function is a response function that should be a dispersive and complex function of frequency so as to satisfy the Kramers-Kronig relations. Certain sum rules [25] for modified spontaneous emission rates, when averaged over all frequencies will therefore not hold in the present formalism that violates these relations. The question is whether dispersion and absorption are important in a particular experiment that one has in mind. Often, optical experiments are interesting only in a frequency range where absorption is indeed negligible, for example, when measuring light emitted by excited atoms inside a photonic crystal. In such cases, it is common practice to neglect material dispersion and absorption in the theoretical description as well $[16,26]$.

Quantum optical descriptions exist where dielectric functions do satisfy the Kramers-Kronig relations, both for homogeneous [27,28] and inhomogeneous dielectrics [29-32]. Usually, the guest atoms are introduced into these theories in the simpler of the two ways described above, as probe atoms with known properties in a two- or three-level description. It would be interesting to introduce guest atoms in theories of inhomogeneous Kramers-Kronig dielectrics in the more fundamental way, starting with Maxwell's equations with the charges of the guest atoms as sources but this will not be done here. Nor will we look at more microscopic descriptions of the dielectric [33-35] where dispersion shows up naturally. It would be very challenging to derive optical predictions from a microscopic description of light and the matter that builds up a complex dielectric such as a photonic crystal.

The goal of this paper is to derive a Hamiltonian with multipolar interaction between the guest atoms and the electromagnetic field inside the inhomogeneous and nondispersive dielectric. The dipole Hamiltonian can then be found as an approximation. First, field quantization of a dielectric without guest atoms is described in Sec. II. Atoms are introduced into the dielectric in Sec. III. The starting point will be Maxwell's equations and a minimal-coupling Lagrangian that produces these equations. Special attention is paid to check whether Maxwell's equations still hold after choosing a gauge. This requires an interesting analysis of functional differentiation after choosing a gauge, presented in Sec. IV. The minimal-coupling Lagrangian is then transformed in Sec. V into its multipolar form with use of the PowerZienau-Woolley transformation that is well known for free space [13]. Our careful analysis of functional differentiation allows us to use a transformation that is simpler and more like the free-space case than presented in related works $[17,18]$. After the transformation, canonical variables and fields are determined. In particular, the important question as to which field in the dielectric is canonically conjugate to the vector potential can be answered more easily than in Refs. $[17,18]$ and the answer will be different than that presented in Ref. [17]. The multipolar Hamiltonian and its dipole approximation are given in second-quantization notation. Results are compared to the free-space case. The free-space dipole-coupling controversy and confusion is reviewed in Sec. VI and the results of this paper are discussed in that perspective. The quantum optics of dielectrics that also have inhomogeneous magnetic properties are briefly discussed in Sec. VII, before concluding in Sec. VIII.

\section{INHOMOGENEOUS DIELECTRIC WITHOUT GUEST ATOMS}

\section{A. Classical Lagrangian and Hamiltonian}

In this section, the quantization of the electromagnetic field in inhomogeneous dielectrics $[15,16]$ is reviewed. The emphasis will be on concepts and results that will be employed in the following sections when guest atoms are introduced in the dielectric.

In SI units, the source-free Maxwell equations in matter are

$$
\begin{array}{cc}
\boldsymbol{\nabla} \cdot \mathbf{B}=0, & \boldsymbol{\nabla} \times \mathbf{E}+\dot{\mathbf{B}}=\mathbf{0}, \\
\boldsymbol{\nabla} \cdot \mathbf{D}=0, & \boldsymbol{\nabla} \times \mathbf{H}-\dot{\mathbf{D}}=\mathbf{0} .
\end{array}
$$

Here, and in the following, the dot denotes a partial time derivative. Fields $\mathbf{E}, \mathbf{B}, \mathbf{D}$, and $\mathbf{H}$ are the electric field and the magnetic induction, the displacement field and the magnetic field vector, respectively. For nonmagnetic inhomogeneous dielectrics, the constitutive relations are simply $\mathbf{B}$ $=\mu_{0} \mathbf{H}$ and $\mathbf{D}=\varepsilon_{0} \varepsilon(\mathbf{r}) \mathbf{E}$. (The generalization of the present theory to inhomogeneous magnetic materials will be discussed in Sec. VII.) As for free space, the electric and magnetic fields can be expressed in terms of a vector potential $\mathbf{A}$ and a scalar potential $\Phi$ :

$$
\mathbf{E}=-\boldsymbol{\nabla} \Phi-\dot{\mathbf{A}}, \quad \mathbf{B}=\boldsymbol{\nabla} \times \mathbf{A} .
$$

There is gauge freedom in choosing pairs $(\mathbf{A}, \Phi)$ that leads to the same electric and magnetic fields. Now choose the generalized Coulomb gauge which is defined by the requirement that the vector potential satisfies

$$
\boldsymbol{\nabla} \cdot[\varepsilon(\mathbf{r}) \mathbf{A}(\mathbf{r})]=0 .
$$

The vector potential or any field satisfying this condition is called "generalized transverse" because it satisfies a generalized version of the Coulomb gauge condition $\boldsymbol{\nabla} \cdot \mathbf{A}=0$ in free space. In the generalized Coulomb gauge, the vector potential must satisfy the wave equation

$$
\boldsymbol{\nabla} \times \boldsymbol{\nabla} \times \mathbf{A}+\frac{\varepsilon(\mathbf{r})}{c^{2}} \ddot{\mathbf{A}}=\mathbf{0}
$$

in order to be consistent with Maxwell Eq. (1b). The scalar potential can be chosen identically zero $(\Phi \equiv 0)$ in the generalized Coulomb gauge. 
Since the goal is to find a quantum optical Hamiltonian in the end, one should start with a Lagrangian formalism for the classical Maxwell fields. From the Lagrangian, the canonical fields and their conjugates can be identified, which will become pairs of noncommuting field operators in a later stage. The principle of least action states that fields (and particle variables, when present) minimize the action [13]; the action is defined as the time-integrated Lagrangian between some initial and final times. The requirement that small variations in the fields do not change the action leads to the EulerLagrange equations for the canonical fields, in our case for the vector potential $[12,13]$ :

$$
\frac{\delta L}{\delta \mathbf{A}}-\frac{d}{d t} \frac{\delta L}{\delta \dot{\mathbf{A}}}=\mathbf{0} .
$$

Here, functional derivatives are denoted with " $\delta$ " and more will be said about them later.

A Lagrangian $L_{0}$ for the electromagnetic field in an inhomogeneous medium is

$$
L_{0}=\int d \mathbf{r} \mathcal{L}_{0} \equiv \frac{1}{2} \int d \mathbf{r}\left[\varepsilon_{0} \varepsilon(\mathbf{r}) \dot{\mathbf{A}}^{2}-\mu_{0}^{-1}(\boldsymbol{\nabla} \times \mathbf{A})^{2}\right] .
$$

The Lagrangian is the spatial integral of Lagrangian density $\mathcal{L}_{0}$ over a large volume $V$ that will eventually be sent to infinity. Subscript " 0 " is used to denote the absence of guest atoms in the dielectric. The vector potential is a canonical field variable and its canonically conjugate field can be found as a functional derivative of the Lagrangian density

$$
\Pi \equiv \frac{\delta L_{0}}{\delta \dot{\mathbf{A}}}=\varepsilon_{0} \varepsilon(\mathbf{r}) \dot{\mathbf{A}}=-\mathbf{D} .
$$

(The functional derivative is used somewhat naively here, but the answer is correct, as a more detailed analysis in Sec. IV will show.) In other words, the field canonically conjugate to the vector potential equals minus of the displacement field, which is a transverse field. Proceeding as for free space [13], one finds that the Euler-Lagrange equation of motion for the vector potential leads to wave equation (4) for the vector potential in the medium. The Hamiltonian is

$$
H_{0}=\int d \mathbf{r}\left(\boldsymbol{\Pi} \cdot \dot{\mathbf{A}}-\mathcal{L}_{0}\right)=\frac{1}{2} \int d \mathbf{r}\left[\frac{\boldsymbol{\Pi}^{2}}{\varepsilon_{0} \varepsilon(\mathbf{r})}+\frac{(\boldsymbol{\nabla} \times \mathbf{A})^{2}}{\mu_{0}}\right] .
$$

This is the Hamiltonian for the classical electromagnetic field in an inhomogeneous dielectric, without guest atoms.

\section{B. Complete sets and quantum Hamiltonian}

For a quantum optical description of the dielectric, the electromagnetic fields can best be expanded in terms of harmonic solutions of wave equation (4). With each of these "true modes" one can associate independent canonical variables for which commutation rules can be given. The set of true modes is not unique. This freedom will be used below to choose a particularly convenient set. For example, in vacuum the true modes are transverse plane waves. For the plane waves, one can choose linear combinations of cosine and sine solutions $\cos (\mathbf{k} \cdot \mathbf{r})$ and $\sin (\mathbf{k} \cdot \mathbf{r})$. The complex exponential $\exp (i \mathbf{k} \cdot \mathbf{r})$ is only one such linear combination.

It will now be argued why the field can be expanded in terms of a set of real mode functions and the orthonormality relations of the modes will be derived. The choice of real modes will simplify the quantization procedure. The reason not to start with complex mode functions is that the latter has associated generalized coordinates and momenta that are not Hermitian. This makes intermediate results more complicated $[16,17,36]$. Real mode functions were also used in Ref. [15], without the motivation given here.

Why does a complete set of real mode functions exist? As before, the electromagnetic fields are assumed to live in large volume $V$. Let $Q$ be an abstract operator in Hilbert space, which has a local representation $\left\langle\mathbf{r}|Q| \mathbf{r}^{\prime}\right\rangle=\delta\left(\mathbf{r}-\mathbf{r}^{\prime}\right) Q(\mathbf{r})$ in configuration space:

$$
Q(\mathbf{r}) \equiv \frac{1}{\sqrt{\varepsilon(\mathbf{r})}} \boldsymbol{\nabla} \times \boldsymbol{\nabla} \times \frac{1}{\sqrt{\varepsilon(\mathbf{r})}} .
$$

Operator $Q$ is Hermitian under the normal inner product. Eigenvalues of $Q$ are $\left(\omega_{\lambda} / c\right)^{2}$ and the $\omega_{\lambda}$ will be called eigenfrequencies. All eigenfunctions $\mathbf{g}_{\lambda}$ of $Q$ have property $\boldsymbol{\nabla} \cdot[\sqrt{\varepsilon(\mathbf{r})} \mathbf{g}(\mathbf{r})]=0$. Label $\lambda$ is understood to count both continua and discrete sets of solutions. The subspace of functions in Hilbert space with the same transversality property is spanned by the eigenfunctions of $Q$. Now let $C$ be the operator which is also local in configuration space, where its action is to take the complex conjugate. The dielectric function in this context must also be viewed as an abstract operator $\varepsilon$ with local representation in configuration space: $\left\langle\mathbf{r}|\varepsilon| \mathbf{r}^{\prime}\right\rangle$ $=\delta\left(\mathbf{r}-\mathbf{r}^{\prime}\right) \varepsilon(\mathbf{r})$. The representations of $Q$ and $C$ commute in configuration space because $\varepsilon(\mathbf{r})$ is real for all positions $\mathbf{r}$ in $V$. Then, $Q$ and $C$ commute in any representation. From the fact that $Q$ and $C$ commute, it follows that an orthonormal basis of real eigenfunctions $\left\{\mathbf{g}_{\lambda}\right\}$ of $Q$ can be chosen to span the subspace (with complex coefficients).

The above analysis shows that vector potential $\mathbf{A}$ can be expanded in terms of a complete set of real vector mode functions $\left\{\mathbf{h}_{\lambda}(\mathbf{r})\right\} \equiv\left\{\sqrt{\varepsilon(\mathbf{r})} \mathbf{g}_{\lambda}(\mathbf{r})\right\}$, which are the harmonic solutions of wave equation (4):

$$
\boldsymbol{\nabla} \times \boldsymbol{\nabla} \times \mathbf{h}_{\lambda}(\mathbf{r})-\frac{\varepsilon(\mathbf{r}) \omega_{\lambda}^{2}}{c^{2}} \mathbf{h}_{\lambda}(\mathbf{r})=\mathbf{0} .
$$

These mode functions satisfy the same generalized transversality condition (3) as the vector potential. As is clear from Eq. (10), unlike $\mathbf{g}_{\lambda}$ functions $\mathbf{h}_{\lambda}$ do not satisfy a Hermitian eigenvalue equation. (To each type of modes corresponds a different density of states [37].) From the orthonormality of $\mathbf{g}_{\lambda}$ it follows that functions $\mathbf{h}_{\lambda}(\mathbf{r})$ satisfy the generalized orthonormality condition $[15,16]$ :

$$
\int d \mathbf{r} \varepsilon(\mathbf{r}) \mathbf{h}_{\lambda}^{*}(\mathbf{r}) \cdot \mathbf{h}_{\lambda^{\prime}}(\mathbf{r})=\delta_{\lambda \lambda^{\prime}}
$$


The complex-conjugation symbol * was written for future reference since, of course, $\mathbf{h}_{\lambda}$ is real. Equation (11) will be called a generalized inner product of modes $\mathbf{h}_{\lambda}$ and $\mathbf{h}_{\lambda^{\prime}}$. The spatial integral in Eq. (11) runs over volume $V$, so that the mode functions scale as $(V)^{-1 / 2}$.

In a scattering situation, where $\varepsilon(\mathbf{r})$ is a space-filling dielectric function plus a local modification within a scattering volume $V_{\mathrm{s}}$, the contribution of the scattering volume to integral (11) scales as $V_{\mathrm{s}} / V$. This fraction becomes zero when quantization volume $V$ is sent to infinity. As an example, mode functions of an infinite photonic crystal with a single point defect (extra or missing dielectric material) have the same orthonormality relations as the mode functions in the absence of the defect.

Functions $\mathbf{h}_{\lambda}$ are complete in the sense that they form a basis for generalized transverse functions (such as the vector potential) that satisfy wave equation (4). In other words, a generalized transverse delta function $\boldsymbol{\delta}_{\varepsilon}^{T}$ (a distribution) can be defined in terms of functions $\mathbf{h}_{\lambda}$ :

$$
\boldsymbol{\delta}_{\varepsilon}^{\mathrm{T}}\left(\mathbf{r}, \mathbf{r}^{\prime}\right) \equiv \sum_{\lambda} \mathbf{h}_{\lambda}(\mathbf{r}) \mathbf{h}_{\lambda}\left(\mathbf{r}^{\prime}\right) \varepsilon\left(\mathbf{r}^{\prime}\right)
$$

For $\varepsilon(\mathbf{r}) \equiv 1$, this expression reduces to the (real) free-space transverse delta function (see Ref. [12], p. 53):

$$
\boldsymbol{\delta}^{\mathrm{T}}(\mathbf{r})=\frac{2}{3} \delta(\mathbf{r}) I-\frac{1}{4 \pi r^{3}}(\mathrm{I}-3 \hat{\boldsymbol{r}} \otimes \hat{\boldsymbol{r}}),
$$

where $I$ is the unit tensor in three dimensions and $\hat{\boldsymbol{r}}$ is the unit vector $\mathbf{r} /|\mathbf{r}|$. Evidently, the generalized transverse delta function (12) is real because the mode functions are real. From its definition (12) and normalization condition (11) of the modes, it follows that $\boldsymbol{\delta}_{\varepsilon}^{T}$ is idempotent:

$$
\int d \mathbf{r}_{1} \boldsymbol{\delta}_{\varepsilon}^{\mathrm{T}}\left(\mathbf{r}, \mathbf{r}_{1}\right) \cdot \boldsymbol{\delta}_{\varepsilon}^{\mathrm{T}}\left(\mathbf{r}_{1}, \mathbf{r}^{\prime}\right)=\boldsymbol{\delta}_{\varepsilon}^{\mathrm{T}}\left(\mathbf{r}, \mathbf{r}^{\prime}\right) .
$$

In other words, $\boldsymbol{\delta}_{\varepsilon}^{\mathrm{T}}$ is a projector into the subspace of generalized transverse functions. The generalized transverse delta function is not symmetric in its arguments because it is transverse in its second and generalized transverse in its first variable:

$$
\boldsymbol{\nabla}_{\mathbf{r}} \cdot\left[\varepsilon(\mathbf{r}) \boldsymbol{\delta}_{\varepsilon}^{\mathrm{T}}\left(\mathbf{r}, \mathbf{r}^{\prime}\right)\right]=0=\nabla_{\mathbf{r}}^{\prime} \cdot\left[\boldsymbol{\delta}_{\varepsilon}^{\mathrm{T}}\left(\mathbf{r}, \mathbf{r}^{\prime}\right)\right] .
$$

The vector potential and its canonically conjugate field have normal-mode expansions:

$$
\begin{aligned}
\mathbf{A}(\mathbf{r}, t) & =1 /\left(\sqrt{\varepsilon_{0}}\right) \sum_{\lambda} q_{\lambda}(t) \mathbf{h}_{\lambda}(\mathbf{r}), \\
\boldsymbol{\Pi}(\mathbf{r}, t) & =\sqrt{\varepsilon_{0}} \sum_{\lambda} p_{\lambda}(t) \varepsilon(\mathbf{r}) \mathbf{h}_{\lambda}(\mathbf{r}),
\end{aligned}
$$

with generalized coordinates $q_{\lambda}(t)$ and momenta $p_{\lambda}(t)$. At this point, the choice of real mode functions pays off because the associated generalized coordinates and momenta are only real when the modes are real; only real coordinates and mo- menta will become Hermitian operators in a quantum description. If the above two expansions are substituted in Hamiltonian (8) and normalization condition (11) is used, then it follows that $H=\frac{1}{2} \Sigma_{\lambda}\left(p_{\lambda}^{2}+\omega_{\lambda}^{2} q_{\lambda}^{2}\right)$. The Hamiltonian turns out to be a simple sum over the true modes of the inhomogeneous dielectric, where the energy of each mode corresponds to a one-dimensional harmonic oscillator with position $q_{\lambda}$, momentum $p_{\lambda}$, and frequency $\omega_{\lambda}$.

Now comes the quantization step. The independent canonical pairs satisfy the standard equal-time commutation relations $\left[q_{\lambda}(t), p_{\lambda^{\prime}}(t)\right]=i \hbar \delta_{\lambda \lambda^{\prime}}$. With normal-mode expansions (16a) and (16b), the commutation relation for the vector potential and its canonically conjugate field can be found immediately:

$$
\left[\mathbf{A}(\mathbf{r}, t), \boldsymbol{\Pi}\left(\mathbf{r}^{\prime}, t\right)\right]=i \hbar \sum_{\lambda} \mathbf{h}_{\lambda}(\mathbf{r}) \mathbf{h}_{\lambda}\left(\mathbf{r}^{\prime}\right) \varepsilon\left(\mathbf{r}^{\prime}\right)=i \hbar \boldsymbol{\delta}_{\varepsilon}^{\mathrm{T}}\left(\mathbf{r}, \mathbf{r}^{\prime}\right)
$$

The commutator is a dyadic quantity. It turns out to be proportional to the generalized transverse delta function.

Annihilation operators are introduced as $\alpha_{\lambda}$ $=\sqrt{\omega_{\lambda} /(2 \hbar)} q_{\lambda}+i \sqrt{1 /\left(2 \hbar \omega_{\lambda}\right)} p_{\lambda}$ and creation operators as their Hermitian conjugates. They have standard commutation relations $\left[\alpha_{\lambda}(t), \alpha_{\lambda^{\prime}}^{\dagger},(t)\right]=\delta_{\lambda \lambda^{\prime}}$ and all other inequivalent commutators are zero. The Hamiltonian becomes the sum over contributions $\hbar \omega_{\lambda}\left(\alpha_{\lambda}^{\dagger} \alpha_{\lambda}+1 / 2\right)$ of individual modes. Thus, the concept of a photon as elementary excitation $\alpha_{\lambda}^{\dagger}|0\rangle$ of a mode is as useful for inhomogeneous dielectrics as it is for free space. Number states, coherent and squeezed states, etc. can be defined analogously. The only difference for inhomogeneous dielectrics is that their true modes are not plane waves.

The vector potential operator and its canonically conjugate field operator can be expressed in terms of creation and annihilation operators as

$$
\begin{gathered}
\mathbf{A}(\mathbf{r}, t)=\sum_{\lambda} \sqrt{\frac{\hbar}{2 \varepsilon_{0} \omega_{\lambda}}}\left[\alpha_{\lambda}(t) \mathbf{h}_{\lambda}(\mathbf{r})+\text { H.c. }\right], \\
\Pi(\mathbf{r}, t)=-i \varepsilon_{0} \varepsilon(\mathbf{r}) \sum_{\lambda} \sqrt{\frac{\hbar \omega_{\lambda}}{2 \varepsilon_{0}}}\left[\alpha_{\lambda}(t) \mathbf{h}_{\lambda}(\mathbf{r})-\text { H.c. }\right] .
\end{gathered}
$$

Here, "H.c." denotes the Hermitian conjugate. The forms of the electric and magnetic fields as quantum-mechanical operators as well as their commutation relations immediately follow from Eq. (2) and the above equation (18a). The time dependence of the operators is simply harmonic, for example, $\alpha_{\lambda}(t)=\alpha(0) \exp \left(-i \omega_{\lambda} t\right)$.

In practice, it can be convenient to use a set of complex true mode functions $\left\{\mathbf{f}_{\mu}\right\}$ instead of real mode functions $\left\{\mathbf{h}_{\lambda}\right\}$. Since the complex mode functions should also satisfy wave equation (10) and the generalized orthonormality condition (11), the two sets of mode functions are related through a unitary transformation $\mathbf{f}_{\mu}=\Sigma_{\lambda} U_{\mu \lambda} \mathbf{h}_{\lambda}$ that only relates mode functions with identical eigenfrequencies; $\mathbf{U}$ is a unitary matrix. Note that because of this unitary relation, the 
generalized transverse delta function (12) can alternatively be expressed in terms of complex mode functions $\mathbf{f}_{\lambda}$. Its effect is the substitution of one $\mathbf{h}_{\lambda}$ by $\mathbf{f}_{\lambda}$ and the other $\mathbf{h}_{\lambda}$ by $\mathbf{f}_{\lambda}^{*}: \boldsymbol{\delta}_{\varepsilon}^{\mathrm{T}}\left(\mathbf{r}, \mathbf{r}^{\prime}\right)$ is also equal to $\Sigma_{\lambda} \mathbf{f}_{\lambda}(\mathbf{r}) \mathbf{f}_{\lambda}^{*}\left(\mathbf{r}^{\prime}\right) \varepsilon\left(\mathbf{r}^{\prime}\right)$. However, after this substitution it is no longer obvious that $\boldsymbol{\delta}_{\varepsilon}^{\mathrm{T}}\left(\mathbf{r}, \mathbf{r}^{\prime}\right)$ is real-valued.

The field operators can also be expanded in terms of the complex mode functions $\mathbf{f}_{\lambda}$ as

$$
\begin{gathered}
\mathbf{A}(\mathbf{r}, t)=\sum_{\lambda} \sqrt{\frac{\hbar}{2 \varepsilon_{0} \omega_{\lambda}}}\left[a_{\lambda}^{(0)}(t) \mathbf{f}_{\lambda}(\mathbf{r})+\text { H.c. }\right], \\
\boldsymbol{\Pi}(\mathbf{r}, t)=-i \varepsilon_{0} \varepsilon(\mathbf{r}) \sum_{\lambda} \sqrt{\frac{\hbar \omega_{\lambda}}{2 \varepsilon_{0}}}\left[a_{\lambda}^{(0)}(t) \mathbf{f}_{\lambda}(\mathbf{r})-\text { H.c. }\right],
\end{gathered}
$$

where the annihilation operator $a_{\mu}^{(0)}$ associated with the complex mode $\mathbf{f}_{\mu}$ is defined in terms of the "old" operators as $a_{\mu}^{(0)} \equiv \Sigma_{\lambda} U_{\mu \lambda}^{-1} \alpha_{\lambda}$ and $a_{\mu}^{(0) \dagger}$ is its Hermitian conjugate. The commutation relations of $a_{\mu}^{(0)}$ and $a_{\mu}^{(0) \dagger}$ are again the standard relations because $\mathbf{U}$ is a unitary transformation. To distinguish $a_{\mu}^{(0)}$ from operators to be defined later, superscript (0) has been added, signifying that no guest atoms are present. The time dependence of the operators is again harmonic.

This completes the quantization of the electromagnetic field in an inhomogeneous dielectric without guest atoms. The reason to start the quantization procedure with real mode functions was that the associated generalized coordinates and momenta are real quantities that become Hermitian operators in quantum mechanics. It is possible to start with complex mode functions instead and to proceed with the nonHermitian operators $[16,17,36]$ but it makes intermediate results unnecessarily more complicated. The unitary relations between complex and real mode functions and between their respective annihilation operators are purely formal unless both sets of mode functions are given explicitly. The above quantization procedure only relies on the mere existence (rather than on an explicit construction) of these unitary mappings.

\section{INHOMOGENEOUS DIELECTRIC WITH GUEST ATOMS}

In the preceding section it was described how to quantize the electromagnetic field in an inhomogeneous dielectric. Now, inside the inhomogeneous dielectric guest atoms are introduced. Their optical response is not included in dielectric function $\varepsilon(\mathbf{r})$ of the medium. The goal in the following sections is to find the quantum optical description of the combined system, with a multipole interaction between the electromagnetic field and the guest atoms. There are at least two reasons why the multipolar Hamiltonian is to be preferred. In the first place, it is more convenient when only approximate calculations can be done, which, in the minimal-coupling formalism, would give gauge-dependent results [11]; second, atoms are much smaller than optical wavelengths and in the multipole formalism this can be exploited well. Actually, atoms are so much smaller than opti- cal wavelengths that often "atoms" are identified with "dipoles."

The starting point is the minimal-coupling Lagrangian that produces the Maxwell equations and the equations of motion for the charges that make up the guest atoms. The minimal-coupling Lagrangian can be used to find a minimalcoupling Hamiltonian and this procedure can be found in Refs. [15,19]. A clear exposition is also given in Ref. [38]. Here, the Lagrangian will first be transformed to the multipolar form before constructing a Hamiltonian. The latter procedure was followed also in Refs. $[17,18]$. The present work is different in some essential aspects that will be stressed where appropriate.

\section{A. Choice of suitable Lagrangian}

Guest atoms inside an inhomogeneous dielectric can be described by a charge density $\sigma_{\mathrm{g}}$ and a current density $\mathbf{J}_{\mathrm{g}}$ which show up as sources in Maxwell's equations [11,17]:

$$
\begin{gathered}
\boldsymbol{\nabla} \cdot \mathbf{B}=0, \\
\boldsymbol{\nabla} \times \mathbf{E}+\dot{\mathbf{B}}=\mathbf{0}, \\
\varepsilon_{0} \boldsymbol{\nabla} \cdot[\varepsilon(\mathbf{r}) \mathbf{E}(\mathbf{r})]=\sigma_{\mathrm{g}}, \\
\mu_{0}^{-1} \boldsymbol{\nabla} \times \mathbf{B}-\varepsilon_{0} \varepsilon(\mathbf{r}) \dot{\mathbf{E}}=\mathbf{J}_{\mathrm{g}} .
\end{gathered}
$$

Here, $\sigma_{\mathrm{g}}$ is the charge density and $\mathbf{J}_{\mathrm{g}}$ the current density produced by the guest atoms alone, as stressed by subscript " $\mathrm{g}$ "; the dielectric is completely described by dielectric function $\varepsilon_{0} \varepsilon(\mathbf{r})$ and magnetic permeability $\mu_{0}$. Whatever Lagrangians and Hamiltonians are introduced for the inhomogeneous dielectric plus guest atoms, they must lead to these four Maxwell equations. Moreover, the electrons with charges $-e$ and masses $m_{\mathrm{e}}$ should respond to electric and magnetic fields as given in the equation of motion

$$
m_{\mathrm{e}} \ddot{\mathbf{r}}_{m j}=-e\left[\mathbf{E}\left(\mathbf{r}_{m j}\right)+\dot{\mathbf{r}}_{m j} \times \mathbf{B}\left(\mathbf{r}_{m j}\right)\right] .
$$

We assume that there are no free charges. All electrons (labeled $j$ ) are bound to atomic nuclei (label $m$ ) to form neutral guest atoms. Then $\sigma_{\mathrm{g}}$ and $\mathbf{J}_{\mathrm{g}}$ are given by [39]

$$
\begin{gathered}
\sigma_{\mathrm{g}}(\mathbf{r}, t)=e \sum_{m}\left[Z_{m} \delta\left(\mathbf{r}-\mathbf{R}_{m}\right)-\sum_{j} \delta\left(\mathbf{r}-\mathbf{r}_{m j}\right)\right], \\
\mathbf{J}_{\mathrm{g}}(\mathbf{r}, t)=-e \sum_{m}\left[\sum_{j} \dot{\mathbf{r}}_{m j} \delta\left(\mathbf{r}-\mathbf{r}_{m j}\right)\right] .
\end{gathered}
$$

Here, $Z_{m}$ is the nuclear charge of atom $m$. The guest atoms are assumed to have fixed positions, their nuclei are their centers of mass and are stationary at positions $\mathbf{R}_{m}$. From these explicit forms of $\sigma_{\mathrm{g}}$ and $\mathbf{J}_{\mathrm{g}}$ follows the equation of continuity or current conservation, $\boldsymbol{\nabla} \cdot \mathbf{J}_{\mathrm{g}}+\dot{\sigma}_{\mathrm{g}}=0$, which can also be found from Maxwell equations (20c) and (20d).

Again, the electric and magnetic fields can be defined through Eq. (2) in terms of a vector potential $\mathbf{A}$ and a scalar potential $\Phi$. Then, the two homogeneous Maxwell equations 
(20a) and (20b) are automatically satisfied. The other two Maxwell equations should follow from the Euler-Lagrange equations [Eq. (5)] for the scalar and the vector potential, respectively. The minimal-coupling Lagrangian is

$$
L_{\text {min }}=\sum_{m, j} \frac{1}{2} m_{\mathrm{e}} \dot{\mathbf{r}}_{m j}^{2}+\int d \mathbf{r} \mathcal{L}_{\min }
$$

Here, Lagrangian density $\mathcal{L}_{\min }$ describes the electromagnetic field energy and its minimal-coupling interaction with the guest atoms:

$$
\mathcal{L}_{\text {min }}=\frac{1}{2} \varepsilon_{0} \varepsilon(\mathbf{r})[\dot{\mathbf{A}}+\boldsymbol{\nabla} \Phi]^{2}-\frac{1}{2 \mu_{0}}(\boldsymbol{\nabla} \times \mathbf{A})^{2}+\mathbf{J}_{\mathrm{g}} \cdot \mathbf{A}-\sigma_{\mathrm{g}} \Phi .
$$

Indeed, Maxwell's third and fourth equations can be found from the Euler-Lagrange equations for the scalar and the vector potentials, respectively. Moreover, the Euler-Lagrange equations for canonical variables $\mathbf{r}_{m j}$ give equations of motion (21) for the charged particles. Note that the Lagrangian leads to these equations of motion, before choosing a gauge to fix $\mathbf{A}$ and $\Phi$ with; the equations of motion are gaugeindependent results that should not depend on the choice of gauge.

\section{B. Fixing the gauge}

The electric and magnetic fields are defined in terms of a scalar and a vector potential. But there is gauge freedom, which means that the scalar and vector potentials are not uniquely defined by the requirement that measurable electric and magnetic fields satisfy Maxwell's equations. We need to choose a gauge in order to find in the end a quantummechanical description of light interacting with the guest atoms. As in the situation without guest atoms in Sec. II, the generalized Coulomb gauge is chosen so that the vector potential satisfies Eq. (3). In this section, it will be checked whether the equations of motion for the scalar and vector potentials still lead to the third and fourth Maxwell equations after choosing the gauge. This must be the case because the choice of gauge should not change the physical predictions of the theory. Still, the check was not performed in Refs. $[17,18]$ and as we shall see, it will be very useful to do so here.

The gauge affects interaction term

$$
L_{\mathbf{A} \Phi} \equiv \varepsilon_{0} \int d \mathbf{r} \varepsilon(\mathbf{r}) \dot{\mathbf{A}} \cdot \nabla \Phi
$$

of Lagrangian (23). The term becomes identically zero because in the generalized Coulomb gauge it has become an inner product of a transverse and a longitudinal function. The remaining terms in the Lagrangian involving the scalar potential lead to an Euler-Lagrange equation that is the generalized Poisson equation for the scalar potential in the Coulomb gauge:

$$
\varepsilon_{0} \boldsymbol{\nabla} \cdot[\varepsilon(\mathbf{r}) \boldsymbol{\nabla} \Phi(\mathbf{r})]=-\sigma_{\mathrm{g}} .
$$

Clearly, the scalar potential cannot be chosen identically zero as in the situation without guest atoms. Gauge-fixing condition (3) for the vector potential and Eq. (26) for the scalar potential, together with the definition of electric field (2) in terms of the two potentials, still lead to the third Maxwell equation (20c).

As in free space, the scalar potential is a function of the positions of the charges that make up the guest atoms [13]. In other words, one can first solve the coupled equations of motion for the vector potential and the charges and from charge distribution $\sigma_{\mathrm{g}}(t)$ thus found, the scalar potential $\Phi(t)$ can be found as the solution of Eq. (26). Therefore, the scalar potential is not an independent canonical field. Lagrangian (23) can then be simplified as

$$
L_{\text {min }}^{\prime}=\sum_{m, j} \frac{1}{2} m_{\mathrm{e}} \dot{\mathbf{r}}_{m j}^{2}-V_{C}+\int d \mathbf{r} \mathcal{L}^{\prime}{ }_{\text {min }},
$$

where Eqs. (25) and (26) were used. Coulomb interaction $V_{C}=\left(\varepsilon_{0} / 2\right) \int d \mathbf{r} \varepsilon(\mathbf{r})(\nabla \Phi)^{2}$ is a function of the guest atoms alone; the Lagrangian density in Eq. (27) becomes

$$
\mathcal{L}^{\prime}{ }_{\text {min }}=\frac{1}{2} \varepsilon_{0} \varepsilon(\mathbf{r}) \dot{\mathbf{A}}^{2}-\frac{1}{2 \mu_{0}}(\boldsymbol{\nabla} \times \mathbf{A})^{2}+\mathbf{J}_{\mathrm{g}} \cdot \mathbf{A} .
$$

Which equation do we find for the vector potential after choosing the generalized Coulomb gauge? Let us begin at the other end; in order to be consistent with the fourth Maxwell equation (20d), the vector potential should satisfy

$$
\mu_{0}^{-1} \boldsymbol{\nabla} \times \boldsymbol{\nabla} \times \mathbf{A}+\varepsilon_{0} \varepsilon(\mathbf{r}) \ddot{\mathbf{A}}=\mathbf{J}_{\mathrm{g}}-\varepsilon_{0} \varepsilon(\mathbf{r}) \boldsymbol{\nabla} \dot{\Phi} .
$$

It is not obvious how source term $-\varepsilon_{0} \varepsilon(\mathbf{r}) \nabla \dot{\Phi}$ can appear at the right-hand side of this equation by functional differentiation of the Lagrangian with respect to the vector potential. Before choosing the gauge, this source term originated from interaction term $L_{\mathrm{A} \Phi}$ [Eq. (25)], which is zero after choosing the gauge. After choosing the generalized Coulomb gauge, a more careful analysis is needed in order to find the fourth Maxwell equation.

\section{FUNCTIONAL DIFFERENTIATION AFTER CHOOSING THE GAUGE}

After choosing the gauge, the vector potential is generalized transverse. The Euler-Lagrange equation for the vector potential is therefore an equation of motion of a constrained system, where the constraint is gauge condition (3). One could try and solve this problem using the method of Lagrange multipliers [40] but this is not the route that will be pursued here. Instead, the appropriate mathematical definition and computation of functional derivatives after choosing a gauge will be studied in the following sections IV A and IV B, respectively. The results will be applied to our physical problem in Sec. IV C.

\section{A. Two definitions of functional derivatives}

Let us generalize the problem somewhat by considering a functional $F=\int d \mathbf{r} \mathcal{F}$ with a functional density $\mathcal{F}$ that de- 
pends on the three-dimensional vector fields $\mathbf{X}$ and $\mathbf{Y}$. Assume also that at some stage, the generalized Coulomb gauge will be chosen for the field $\mathbf{Y}$. This gauge is defined by the requirement that $\boldsymbol{\nabla}_{\mathbf{r}} \cdot[\varepsilon(\mathbf{r}) \mathbf{Y}(\mathbf{r})]$ equals zero.

Before choosing the gauge, the functional derivative of functional $F$ with respect to vector field $\mathbf{Y}$ is defined as

$$
\frac{\delta F}{\delta \mathbf{Y}(\mathbf{r})} \equiv \lim _{\gamma \rightarrow 0} \frac{\int d \mathbf{r}^{\prime}\left\{\mathcal{F}\left[\mathbf{Y}\left(\mathbf{r}^{\prime}\right)+\gamma \delta\left(\mathbf{r}-\mathbf{r}^{\prime}\right) l\right]-\mathcal{F}\left[\mathbf{Y}\left(\mathbf{r}^{\prime}\right)\right]\right\}}{\gamma} .
$$

(The $\mathbf{X}$-dependence of $\mathcal{F}$ was dropped for brevity.) The functional derivative of $F$ with respect to $\mathbf{Y}$ describes the relative changes of $F$ when small variations proportional to $\delta(\mathbf{r}$ $\left.-\mathbf{r}^{\prime}\right) \mid$ are added to vector function $\mathbf{Y}$. Here, $\delta\left(\mathbf{r}-\mathbf{r}^{\prime}\right)$ is the Dirac delta function in three dimensions; as before, $I$ is the unit tensor. It turns out that the right-hand side of Eq. (30) can be computed as the partial derivative of functional density $\mathcal{F}$ with respect to $\mathbf{Y}$. While doing this, the $\mathcal{F}$ can simply be considered as a function and $\mathbf{Y}$ as one of its variables. Before choosing the generalized Coulomb gauge, Eq. (30) correctly defines the functional derivative of $F$ with respect to $\mathbf{Y}$.

Now suppose for the moment that $F$ is defined as $F$ $=\int d \mathbf{r X} \cdot \mathbf{Y}$. Suppose also that field $\mathbf{X}$ is the product of $\varepsilon(\mathbf{r})$ with some longitudinal vector field. Then, $F$ becomes identically zero in the generalized Coulomb gauge because it is the inner product of a transverse and a longitudinal vector field. Still, functional derivative Eq. (30) of $F$ would give a nonzero answer. This can only mean that Eq. (30) does not define the functional derivative with respect to generalized transverse functions correctly. The reason is that the function space in which field $\mathbf{Y}$ lives has become smaller by choosing the gauge; it now lives in the subspace of functions that are generalized transverse. This also means that functional variations of $\mathbf{Y}$ should stay inside this subspace. In functional derivative (30), variations in the whole function space are allowed and clearly, $\boldsymbol{\nabla}_{\mathbf{r}^{\prime}} \cdot\left[\varepsilon\left(\mathbf{r}^{\prime}\right) \delta\left(\mathbf{r}-\mathbf{r}^{\prime}\right) \mid\right]$ is nonzero.

In general, with every set of constraints a new functional derivative can be associated. Here, only the gauge constraint will be considered that functions be generalized transverse. Functional differentiation with respect to generalized transverse functions can be defined as (see [36], p. 20)

$$
\frac{\delta F}{\delta \mathbf{Y}_{\varepsilon}^{\mathrm{T}}(\mathbf{r})} \equiv \lim _{\gamma \rightarrow 0} \frac{\int d \mathbf{r}^{\prime}\left\{\mathcal{F}\left[\mathbf{Y}\left(\mathbf{r}^{\prime}\right)+\gamma \boldsymbol{\delta}_{\varepsilon}^{\mathrm{T}}\left(\mathbf{r}^{\prime}, \mathbf{r}\right)\right]-\mathcal{F}\left[\mathbf{Y}\left(\mathbf{r}^{\prime}\right)\right]\right\}}{\gamma}
$$

with the generalized transverse delta function $\boldsymbol{\delta}_{\varepsilon}^{T}$ as defined in Eq. (12). In this functional derivative, the functional variations do stay inside the generalized transverse subspace since $\boldsymbol{\delta}_{\varepsilon}^{\mathrm{T}}$ is the projector into the subspace and $\boldsymbol{\nabla}_{\mathbf{r}^{\prime}} \cdot\left[\varepsilon\left(\mathbf{r}^{\prime}\right) \boldsymbol{\delta}_{\varepsilon}^{\mathrm{T}}\left(\mathbf{r}^{\prime}, \mathbf{r}\right)\right]=0$. Derivative (31) will be called the "constrained functional derivative" in the following.

\section{B. Simple rules to compute constrained functional derivatives}

Now the goal is to find simple rules to compute the constrained functional derivative (31) with respect to generalized transverse functions, just like the normal functional derivative (30) can simply be calculated as a partial derivative. With that goal in mind, first some properties of generalized transverse functions are derived.

With every transverse function $\mathbf{X}^{\mathrm{T}}(\mathbf{r})$, a generalized transverse function $\left[\mathbf{X}^{\mathrm{T}}(\mathbf{r}) / \varepsilon(\mathbf{r})\right]$ can be associated. From section II B we know that the latter function has an expansion in terms of generalized transverse modes $\mathbf{h}_{\lambda}(\mathbf{r})$, so that $\mathbf{X}^{\mathrm{T}}(\mathbf{r})$ can be expanded in terms of $\varepsilon(\mathbf{r}) \mathbf{h}_{\lambda}(\mathbf{r})$. This simple fact, in combination with Eqs. (11) and (12), leads to the following projection properties of $\boldsymbol{\delta}_{\varepsilon}^{\mathrm{T}}$ :

$$
\begin{gathered}
\int d \mathbf{r}^{\prime} \mathbf{X}^{\mathrm{T}}\left(\mathbf{r}^{\prime}\right) \cdot \boldsymbol{\delta}_{\varepsilon}^{\mathrm{T}}\left(\mathbf{r}^{\prime}, \mathbf{r}\right)=\mathbf{X}^{\mathrm{T}}(\mathbf{r}), \\
\varepsilon(\mathbf{r}) \int d \mathbf{r}^{\prime} \boldsymbol{\delta}_{\varepsilon}^{\mathrm{T}}\left(\mathbf{r}, \mathbf{r}^{\prime}\right) \cdot \mathbf{X}^{\mathrm{T}}\left(\mathbf{r}^{\prime}\right) / \varepsilon\left(\mathbf{r}^{\prime}\right)=\mathbf{X}^{\mathrm{T}}(\mathbf{r}), \\
\int d \mathbf{r}^{\prime} \boldsymbol{\delta}_{\varepsilon}^{\mathrm{T}}\left(\mathbf{r}, \mathbf{r}^{\prime}\right) \cdot \mathbf{X}^{\mathrm{L}}\left(\mathbf{r}^{\prime}\right)=\mathbf{0}, \\
\int d \mathbf{r}^{\prime} \varepsilon\left(\mathbf{r}^{\prime}\right) \mathbf{X}^{\mathrm{L}}\left(\mathbf{r}^{\prime}\right) \cdot \boldsymbol{\delta}_{\varepsilon}^{\mathrm{T}}\left(\mathbf{r}^{\prime}, \mathbf{r}\right)=\mathbf{0},
\end{gathered}
$$

for any transverse function $\mathbf{X}^{\mathrm{T}}$ (zero divergence) and longitudinal function $\mathbf{X}^{\mathrm{L}}$ (zero curl).

With the use of Eqs. (32a) and (32d), functional derivative (31) is simple in the following two important cases:

$$
\begin{gathered}
\frac{\delta}{\delta \mathbf{Y}_{\varepsilon}^{\mathrm{T}}(\mathbf{r})} \int d \mathbf{r}^{\prime} \mathbf{X}^{\mathrm{T}}\left(\mathbf{r}^{\prime}\right) \cdot \mathbf{Y}\left(\mathbf{r}^{\prime}\right)=\mathbf{X}^{\mathrm{T}}(\mathbf{r}), \\
\frac{\delta}{\delta \mathbf{Y}_{\varepsilon}^{\mathrm{T}}(\mathbf{r})} \int d \mathbf{r}^{\prime} \varepsilon\left(\mathbf{r}^{\prime}\right) \mathbf{X}^{\mathrm{L}}\left(\mathbf{r}^{\prime}\right) \cdot \mathbf{Y}\left(\mathbf{r}^{\prime}\right)=\mathbf{0},
\end{gathered}
$$

where $\mathbf{X}^{\mathrm{T}}$ and $\mathbf{X}^{\mathrm{L}}$ are arbitrary transverse and longitudinal functions, respectively. The second case (33b) makes clear that the constrained functional derivative of inner products of transverse and longitudinal fields indeed gives zero; the first case (33a) shows that the partial-derivative-of- $\mathcal{F}$ computation rule still gives the correct answers for inner products of $\mathbf{Y}_{\varepsilon}^{\mathrm{T}}$ with transverse functions.

How can the constrained functional derivative be calculated in the more general situation

$$
\frac{\delta}{\delta \mathbf{Y}_{\varepsilon}^{\mathrm{T}}(\mathbf{r})} \int d \mathbf{r}^{\prime} \mathbf{X}\left(\mathbf{r}^{\prime}\right) \cdot \mathbf{Y}\left(\mathbf{r}^{\prime}\right)
$$

where $\mathbf{X}$ is a general vector function? It will now be shown that any vector field $\mathbf{X}$ can be decomposed such that the only two rules of computation needed are the simple cases (33a) and (33b). 
Given vector field $\mathbf{X}$, construct scalar field $\sigma_{\mathbf{X}} \equiv-\boldsymbol{\nabla} \cdot \mathbf{X}$. Now, find potential $\chi$, given "charge distribution" $\sigma_{\mathbf{X}}$ and dielectric function $\varepsilon_{0} \varepsilon(\mathbf{r})$, from the following generalized Poisson equation:

$$
\varepsilon_{0} \boldsymbol{\nabla} \cdot[\varepsilon(\mathbf{r}) \boldsymbol{\nabla} \chi(\mathbf{r})]=-\sigma_{\mathbf{X}}(\mathbf{r}) .
$$

This is a well-known problem in electrostatics. There is a unique solution for $\chi$ of this inhomogeneous problem, given the charge distribution and the boundary condition that the potential be zero at infinity. With potential $\chi$ thus found, define two vector fields $\mathbf{X}_{1}$ and $\mathbf{X}_{2}$ as

$$
\begin{gathered}
\mathbf{X}_{1} \equiv \mathbf{X}(\mathbf{r})-\varepsilon_{0} \varepsilon(\mathbf{r}) \boldsymbol{\nabla} \chi(\mathbf{r}), \\
\mathbf{X}_{2} \equiv \varepsilon_{0} \varepsilon(\mathbf{r}) \boldsymbol{\nabla} \chi(\mathbf{r}),
\end{gathered}
$$

so that evidently, $\mathbf{X}=\mathbf{X}_{1}+\mathbf{X}_{2}$. Vector field $\mathbf{X}_{1}$ is transverse by construction of potential $\chi$; field $\left[\mathbf{X}_{2} / \varepsilon(\mathbf{r})\right]$ is of course longitudinal. In summary, the following theorem was proven: An arbitrary vector field can be uniquely decomposed into a part which, after division by $\varepsilon(\mathbf{r})$ is longitudinal and a transverse part. This theorem is useful for evaluating the constrained functional derivative because it leads to

$$
\frac{\delta}{\delta \mathbf{Y}_{\varepsilon}^{\mathrm{T}}(\mathbf{r})} \int d \mathbf{r}^{\prime} \mathbf{X}\left(\mathbf{r}^{\prime}\right) \cdot \mathbf{Y}\left(\mathbf{r}^{\prime}\right)=\mathbf{X}(\mathbf{r})-\varepsilon_{0} \varepsilon(\mathbf{r}) \boldsymbol{\nabla} \chi(\mathbf{r}),
$$

where the unique decomposition of $\mathbf{X}$ was used and the simple derivatives Eqs. (33a) and (33b) were applied to $\mathbf{X}_{1}$ and $\mathbf{X}_{2}$, respectively. The problem of computing a functional derivative with respect to a generalized transverse function has thus been reduced to a problem in electrostatics. Note that the constrained functional derivative (37) produces a field that is always transverse. That transverse field is equal to the transverse part of $\mathbf{X}$ if $\mathbf{X}$ itself is transverse or if $\varepsilon(\mathbf{r}) \equiv 1$.

The unique decompositions (36a) and (36b) of vector fields is a generalization of the Helmholtz theorem [41] but the name "generalized Helmholtz theorem" was already given to a slightly different statement [18], namely: Every vector field $\mathbf{Z}$ can be uniquely decomposed as the sum of a generalized transverse vector field $\mathbf{Z}_{1}$ and a longitudinal field $\mathbf{Z}_{2}$. (The proof of this theorem in Ref. [18] begs the question whether the part of the decomposition that is called longitudinal indeed has zero curl but one can show that this is the case.) As a corollary of decompositions (36a) and (36b), another short proof can be given of the generalized Helmholtz theorem. The proof is simple: given $\mathbf{Z}$, define $\mathbf{X}$ $=\varepsilon(\mathbf{r}) \mathbf{Z}$. Then, apply the previous unique decomposition to $\mathbf{X}$, as in Eqs. (36a) and (36b). Define $\mathbf{Z}_{1} \equiv \mathbf{X}_{1} / \varepsilon(\mathbf{r})$ and $\mathbf{Z}_{2}$ $\equiv \mathbf{X}_{2} / \varepsilon(\mathbf{r})$. Then, it follows that $\mathbf{Z}=\mathbf{Z}_{1}+\mathbf{Z}_{2}$, where $\mathbf{Z}_{1}$ is a generalized transverse and $\mathbf{Z}_{2}$ is a longitudinal field. This completes the proof.

\section{Functional derivatives of the minimal-coupling Lagrangian}

The definition of the constrained functional derivative and its computation rules can now be applied to our case of in- terest, where the functional is Lagrangian $L_{\text {min }}^{\prime}$, Eq. (27), and where the generalized Coulomb gauge applies to vector potential A.

Before choosing the gauge, the "ordinary" functional derivative (30) of the Lagrangian of Eq. (24) with respect to A leads to the fourth Maxwell equation (20d), as it should. After choosing the gauge, interaction term $L_{\mathbf{A} \Phi}$ [Eq. (25)] in the Lagrangian becomes identically zero. Its functional derivative with respect to $\mathbf{A}$ should also be zero. This is indeed the case because constrained derivative (31) is the correct one to use rather than ordinary functional derivative (30) after choosing the gauge. Note that the constrained functional derivative must also be used for free space after choosing the Coulomb gauge, with $\boldsymbol{\delta}_{\varepsilon}^{T}$ equal to $\boldsymbol{\delta}^{T}$ [Eq. (13)]. For free space, the machinery of functional derivatives usually is not introduced and the derivative is taken implicitly, for example, in Ref. [13], p. 289.

The naive calculation of the canonical field in Eq. (7) of Sec. II can now be justified:

$$
\frac{\delta}{\delta \dot{\mathbf{A}}_{\varepsilon}^{\mathrm{T}}(\mathbf{r})} \int d \mathbf{r}^{\prime} \varepsilon\left(\mathbf{r}^{\prime}\right) \dot{\mathbf{A}}^{2}\left(\mathbf{r}^{\prime}\right)=2 \varepsilon(\mathbf{r}) \dot{\mathbf{A}}(\mathbf{r}) .
$$

One can find this result by realizing that the functional on the left-hand side is a special case of Eq. (33a) with fields $\mathbf{X}$ and $\mathbf{Y}$ equal to $\varepsilon(\mathbf{r}) \dot{\mathbf{A}}$ and $\dot{\mathbf{A}}$, respectively.

In the special case that vector field $\mathbf{X}$ is current density $\mathbf{J}_{\mathrm{g}}$ produced by the guest atoms in the dielectric, current conservation implies that scalar field $\sigma_{\mathbf{J}_{\mathrm{g}}}$, as constructed in Sec. IV B equals the time derivative of physical charge density $\sigma_{\mathrm{g}}$. By the uniqueness of the solution of the generalized Poisson equation, potential $\chi$ must then be identified with the time derivative of physical scalar potential $\Phi$. Therefore, the constrained functional derivative of $\int d \mathbf{r} \mathbf{J}_{\mathrm{g}} \cdot \mathbf{A}$ can now be computed as

$$
\frac{\delta}{\delta \mathbf{A}_{\varepsilon}^{\mathrm{T}}(\mathbf{r})} \int d \mathbf{r}^{\prime} \mathbf{J}_{\mathrm{g}}\left(\mathbf{r}^{\prime}\right) \cdot \mathbf{A}\left(\mathbf{r}^{\prime}\right)=\mathbf{J}_{\mathrm{g}}(\mathbf{r})-\varepsilon_{0} \varepsilon(\mathbf{r}) \nabla \dot{\Phi}(\mathbf{r}) .
$$

With this result, the Euler-Lagrange equation for the vector potential from Lagrangian (27) precisely becomes Eq. (29) for the vector potential that we were looking for. Only by the careful computation of functional derivatives, as presented in Secs. IV A and IV B, one can prove that the fourth Maxwell equation (20d) also holds after choosing the generalized Coulomb gauge. Interestingly, before choosing the gauge, the source term $-\varepsilon_{0} \varepsilon(\mathbf{r}) \nabla \dot{\Phi}$ in Eq. (29) came from the $L_{\mathbf{A} \Phi}$ interaction term (25) in the Lagrangian. After choosing the gauge, however, the source term is produced by the constrained functional derivative of the minimal-coupling interaction term $\int d \mathbf{r} \mathbf{J}_{\mathrm{g}} \cdot \mathbf{A}$.

The left-hand side of wave equation (29) is certainly transverse in the generalized Coulomb gauge. The right-hand side is also transverse. A mathematical reason is that the wave equation is found by functional differentiation with respect to generalized transverse functions. In Sec. IV B it was 
shown that these derivatives are always transverse. Physically, the source term must be transverse because of current conservation. [Use Eq. (26).]

\section{THE QUANTUM MULTIPOLAR INTERACTION HAMILTONIAN}

In the preceding sections it was shown that the minimalcoupling Lagrangian produces the Maxwell-Lorentz equations for the electromagnetic fields and the guest charges, before and also after choosing the generalized Coulomb gauge. Now the goal is to transform the gauge-dependent minimal-coupling Lagrangian (27) in order to obtain a Lagrangian with multipole interaction between the electromagnetic field and the guest atoms. The multipolar Lagrangian must lead to the same equations for the fields and charges, of course. Candidate transformations are transformations where a total time derivative of a function of the canonical variables is added to the Lagrangian. Such transformations leave the action unchanged [13]. A particular transformation of this sort will be used shortly but first some fields must be introduced.

\section{A. Polarization, magnetization, and displacement fields}

In the following, it is useful to describe the guest atoms in terms of a polarization density $\mathbf{P}_{\mathrm{g}}$ and a magnetization density $\mathbf{M}_{\mathrm{g}}$, rather than in terms of the charge and current densities. The former and latter pairs are related through [39]:

$$
\sigma_{\mathrm{g}}=-\boldsymbol{\nabla} \cdot \mathbf{P}_{\mathrm{g}}, \quad \mathbf{J}_{\mathrm{g}}=\dot{\mathbf{P}}_{\mathrm{g}}+\boldsymbol{\nabla} \times \mathbf{M}_{\mathrm{g}} .
$$

In terms of the introduced variables, the equation of continuity is automatically satisfied. The polarization and magnetization fields have the following integral representations [39]:

$$
\begin{aligned}
\mathbf{P}_{\mathrm{g}}(\mathbf{r}, t)= & -e \sum_{m j} \int_{0}^{1} d u\left(\mathbf{r}_{m j}-\mathbf{R}_{m}\right) \\
& \times \delta\left(\mathbf{r}-\mathbf{R}_{m}-u\left(\mathbf{r}_{m j}-\mathbf{R}_{m}\right)\right), \\
\mathbf{M}_{\mathrm{g}}(\mathbf{r}, t)= & -e \sum_{m j} \int_{0}^{1} d u u\left(\mathbf{r}_{m j}-\mathbf{R}_{m}\right) \\
& \times \dot{\mathbf{r}}_{m j} \delta\left(\mathbf{r}-\mathbf{R}_{m}-u\left(\mathbf{r}_{m j}-\mathbf{R}_{m}\right)\right) .
\end{aligned}
$$

These polarization and magnetization fields are simply the sums of fields $\mathbf{P}_{\mathrm{g} m}$ and $\mathbf{M}_{\mathrm{g} m}$ produced by the individual guest atoms. Finite-order multipole expansions of the polarization and magnetization fields can be found by truncating the Taylor expansion in $u$ of the integrands on the right-hand sides of the above equations. Such approximations will be made in Sec. V D.

Displacement field $\mathbf{D}$ and magnetic-field vector $\mathbf{H}$ are given by the following constitutive relations:

$$
\mathbf{D} \equiv \varepsilon_{0} \varepsilon(\mathbf{r}) \mathbf{E}+\mathbf{P}_{\mathrm{g}}, \quad \mathbf{H} \equiv \mu_{0}^{-1} \mathbf{B}-\mathbf{M}_{\mathrm{g}} .
$$

Electric field $\mathbf{E}$ and magnetic field $\mathbf{B}$ are again defined by Eq. (2) in terms of a scalar potential $\Phi$ and a vector potential $\mathbf{A}$.
It was assumed as before that the dielectric is nonmagnetic so that the magnetic permeability equals value $\mu_{0}$ of free space. Note that dielectric function $\varepsilon(\mathbf{r})$ is a property of the dielectric alone, independent of the guest atoms. On the other hand, displacement field (42) does depend on the guest atoms because it includes the polarization field produced by them. The displacement field defined here is therefore different (although the same symbol is used) from the displacement field that was defined in Sec. II A, where no guest atoms were present. In that case, the transversality of $\mathbf{D}$ was evident. The displacement field is also transverse when guest atoms are present, according to Eqs. (20c) and (40) in combination with Eq. (42).

\section{B. Classical multipolar Lagrangian and Hamiltonian}

Minimal-coupling Lagrangian (27) will now be transformed to a multipolar Lagrangian by adding to it the total time derivative

$$
-\frac{d}{d t} \int d \mathbf{r} \mathbf{P}_{\mathrm{g}}(\mathbf{r}, t) \cdot \mathbf{A}(\mathbf{r}, t)
$$

This is the Power-Zienau-Woolley (PZW) transformation and its effect is well known for free space [13]. The reason to choose this transformation will be given a posteriori, when discussing the multipolar Hamiltonian. The difference with the free-space PZW transformation is that now the vector potential satisfies the generalized rather than the usual Coulomb gauge. The transformation was already applied to inhomogeneous dielectrics before, in Ref. [17]. There, it was stated that polarization density $\mathbf{P}_{\mathrm{g}}$ in the Lagrangian density should be replaced by a "reduced polarization density" at this point, in order to stick to the generalized Coulomb gauge for the vector potential. However, as will be clear shortly, such replacements are not necessary if functional derivatives with respect to the generalized transverse vector potential are identified as constrained functional differentiations and if computation rules as presented in Sec. IV are used accordingly.

After adding term (43), Lagrangian $L_{\text {multi }}$ can be rewritten as

$$
L_{\text {multi }}=\sum_{m j} \frac{1}{2} m_{\mathrm{e}} \dot{\mathbf{r}}_{m j}^{2}-V_{C}+\int d \mathbf{r} \mathcal{L}_{\text {multi }}
$$

Lagrangian density $\mathcal{L}_{\text {multi }}$ has form

$$
\mathcal{L}_{\text {multi }}=\frac{1}{2} \varepsilon_{0} \varepsilon(\mathbf{r}) \dot{\mathbf{A}}^{2}-\frac{1}{2 \mu_{0}}(\boldsymbol{\nabla} \times \mathbf{A})^{2}+\mathbf{M}_{\mathrm{g}} \cdot \boldsymbol{\nabla} \times \mathbf{A}-\mathbf{P}_{\mathrm{g}} \cdot \dot{\mathbf{A}}
$$

where the definition of the magnetization density (41b) was used as well as Gauss's theorem. The derivation is identical to the free-space case.

In order to find a Hamiltonian, first the canonically conjugate variables must be determined. By reasoning as in Sec. IV $\mathrm{C}$, the constrained functional differentiation of Lagrangian $L_{\text {multi }}$ with respect to $\dot{\mathbf{A}}$ produces the following field that is canonically conjugate to the vector potential: 


$$
\boldsymbol{\Pi} \equiv \frac{\delta L_{\text {multi }}}{\delta \dot{\mathbf{A}}_{\varepsilon}^{\mathrm{T}}}=\varepsilon_{0} \varepsilon(\mathbf{r}) \dot{\mathbf{A}}-\left[\mathbf{P}_{\mathrm{g}}-\varepsilon_{0} \varepsilon(\mathbf{r}) \nabla \Phi\right]=-\mathbf{D} .
$$

Here, the definition of electric field (2) and displacement field (42) were used. As in the case without guest atoms, the field canonically conjugate to the vector potential equals minus of the displacement field. The difference is that now the displacement field also contains the polarization field produced by the guest atoms. The canonically conjugate field would have been different if the minimal-coupling Lagrangian had been used.

The resulting Eq. (46) that the canonically conjugate field of the vector potential is the full displacement field of the medium including guest atoms, is an important generalization of the free-space result [13]. In our formalism, it could be found rather easily, by realizing that functional derivatives must be redefined after choosing a gauge. In Ref. [17], a canonically conjugate field was identified, which was stated to be different from the displacement field; in Ref. [18], the matter was reconsidered and the displacement field was found as the canonically conjugate field after all but only because polarization field $\mathbf{P}_{\mathrm{g}}$ in PZW transformation (43) was replaced by a reduced polarization field for reasons that remain somewhat unclear. The effect of the replacement seems to be that functional derivatives with respect to the vector potential can be calculated as partial derivatives, a computation rule that in general is valid only before choosing the gauge. In contrast, our PZW transformation (43) features the usual polarization field of the guest atoms, whether we choose to do the transformation before or after fixing the gauge. We think that our approach is more transparent and more widely applicable.

Canonical momenta $\mathbf{p}_{m j}$ corresponding to coordinate variables $\mathbf{q}_{m j}$ of the guest charges are

$$
\mathbf{p}_{m j}=m_{\mathrm{e}} \dot{\mathbf{r}}_{m j}-\mathbf{F}_{m j}
$$

where field $\mathbf{F}_{m j}$ stems from magnetization density (41b) and is defined as

$$
\mathbf{F}_{m j} \equiv e \int_{0}^{1} d u u \mathbf{B}\left[\mathbf{R}_{m}-u\left(\mathbf{r}_{m j}-\mathbf{R}_{m}\right)\right] \times\left(\mathbf{r}_{m j}-\mathbf{R}_{m}\right) .
$$

Note that unlike $\mathbf{A}$ and $\mathbf{q}_{m j}$, their canonically conjugate variables $\boldsymbol{\Pi}$ and $\mathbf{p}_{m j}$ are not fully electromagnetic or fully atomic in nature, respectively.

All canonical momenta have now been determined so that the multipolar Hamiltonian $H_{\text {multi }}$ can be given in terms of the canonical variables $\left(\mathbf{r}_{m j}, \mathbf{p}_{m j}\right)$ and canonical fields (A, II):

$$
\begin{gathered}
H_{\text {multi }}=\sum_{m j} \mathbf{p}_{m j} \cdot \dot{\mathbf{r}}_{m j}+\int d \mathbf{r} \boldsymbol{\Pi} \cdot \dot{\mathbf{A}}-L_{\text {multi }} \\
=H_{\mathrm{rad}}+H_{\mathrm{at}}+\sum_{m}\left[V_{\mathrm{P}}^{(m)}+V_{\mathrm{M}}^{(m)}\right] .
\end{gathered}
$$

The total Hamiltonian consist of a radiative and an atomic part, plus electric and magnetic interactions between field and matter. The radiative part of the Hamiltonian is

$$
H_{\mathrm{rad}}=\int d \mathbf{r}\left[\frac{\boldsymbol{\Pi}^{2}}{2 \varepsilon_{0} \varepsilon(\mathbf{r})}+\frac{\mathbf{B}^{2}}{2 \mu_{0}}\right]
$$

consisting of an electric and magnetic field-energy term, respectively. The form of the radiative Hamiltonian has not changed after adding the guest atoms but there is a slight shift in its interpretation since the polarization of the atoms is included in the conjugate field. The atomic polarization field of atom $m$ interacts with the electromagnetic field as described by

$$
V_{\mathrm{P}}^{(m)}=\int d \mathbf{r} \frac{\mathbf{P}_{\mathrm{g} m} \cdot \boldsymbol{\Pi}}{\varepsilon_{0} \varepsilon(\mathbf{r})} .
$$

In most cases, this is the dominant interaction between field and matter. The usually weaker magnetic interaction consists of two terms:

$$
V_{\mathbf{M}}^{(m)}=-\mathbf{M}_{\mathrm{g} m}^{\prime} \cdot \mathbf{B}+\sum_{m j} \frac{F_{m j}^{2}}{2 m_{\mathrm{e}}} .
$$

The first term is linear in the magnetic field and represents the paramagnetic energy. Instead of magnetization $\mathbf{M}_{\mathbf{g} m}$, a reduced magnetization $\mathbf{M}_{\mathrm{g} m}^{\prime}$ has been used in this first term. The reduced magnetization is defined as the magnetization [see Eq. (41b)] with the $\dot{\mathbf{r}}_{m j}$ replaced by $\mathbf{p}_{m j} / m_{\mathrm{e}}[13,17]$. The difference has been corrected for by a sign change of the second term, which is quadratic in the magnetic field. This is the diamagnetic energy of the guest atoms in the nonmagnetic dielectric. It can be safely ignored from now on since it is much smaller than the other two interactions (see Ref. [11], Sec. 8.6).

Only the atomic part Hamiltonian (50) must still be discussed. It has form

$$
\sum_{m j} \frac{p_{m j}^{2}}{2 m_{\mathrm{e}}}+\int d \mathbf{r} \frac{\mathbf{P}_{\mathrm{g}}^{2}}{2 \varepsilon_{0} \varepsilon(\mathbf{r})}
$$

The first term in the atomic Hamiltonian (54) represents the kinetic energy of the guest charges; the second term is the potential energy of the guest atoms, expressed as a polarization energy. The Coulomb term $V_{C}$ is absent in the Hamiltonian, because it cancels against the other term quadratic in $\boldsymbol{\nabla} \Phi$ which one gets when solving Eq. (46) for $\dot{\mathbf{A}}$ and substituting the result in Hamiltonian (50).

It is natural to split the polarization energy in Eq. (50) into an intra-atomic and an interatomic polarization energy, respectively $[13,18]$ :

$$
\int d \mathbf{r} \frac{\mathbf{P}_{\mathrm{g}}^{2}}{2 \varepsilon_{0} \varepsilon(\mathbf{r})}=\sum_{m} \int d \mathbf{r} \frac{P_{\mathrm{g} m}^{2}}{2 \varepsilon_{0} \varepsilon(\mathbf{r})}+\sum_{m \neq n} \int d \mathbf{r} \frac{\mathbf{P}_{\mathrm{g} m} \cdot \mathbf{P}_{\mathrm{g} n}}{\varepsilon_{0} \varepsilon(\mathbf{r})} .
$$

The intra-atomic polarization energy is the potential energy that keeps an atom together; the interatomic polarization energy is the only interaction term between neutral atoms in 
multipolar Hamiltonian (50). The Hamiltonian is still classical, so that polarization field $\mathbf{P}_{\mathrm{g} m}(\mathbf{r})$ [Eq. (41a)] is identically zero outside the smallest sphere surrounding all charges that make up (neutral) atom $m$. For that reason, the classical interatomic polarization energy (also known as contact energy) is identically zero unless bounding spheres of distinct guest atoms overlap. In the quantum-mechanical description that will be given shortly, the expectation value of the polarization energy will not be identically zero. The atomic wave functions of distinct guest atoms have a nonvanishing overlap. However, the overlap falls off exponentially with interatomic distance and is negligible unless the distance is of the order of the size of the atoms.

In the rest of this paper, the guest atoms are assumed more than a few nanometers apart and their contact energies are neglected. Then, the atomic Hamiltonian for all guest atoms is simply the sum of single-atom Hamiltonians

$$
H_{\mathrm{at}}^{(m)}=\sum_{j} \frac{p_{m j}^{2}}{2 m_{\mathrm{e}}}+\int d \mathbf{r} \frac{\mathbf{P}_{\mathrm{g} m}^{2}}{2 \varepsilon_{0} \varepsilon(\mathbf{r})}
$$

The total Hamiltonian Eq. (50) becomes

$$
H_{\text {multi }}=H_{\mathrm{rad}}+\sum_{m}\left[H_{\mathrm{at}}^{(m)}+V_{\mathrm{P}}^{(m)}+V_{\mathrm{M}}^{(m)}\right] .
$$

Just like in the free-space case [13], in multipole Hamiltonian (57) for inhomogeneous dielectrics, there is no instantaneous interaction term left between well-separated neutral guest atoms. This means that in the multipolar representation, atoms notice each other only because they interact with the same (retarded) electromagnetic fields $\mathbf{D} /\left[\varepsilon_{0} \varepsilon(\mathbf{r})\right]$ and B. Of course, the multipolar and the minimal-coupling representations should give identical physical predictions; in Refs. $[12,13]$ the equivalence is proved for several observables in free space. The absence of direct interatomic interactions often makes calculations simpler in the multipolar representation. This justifies the choice of PZW transformation (43) out of many candidate transformations.

\section{Quantum multipolar Hamiltonian}

The goal is now to rewrite Hamiltonian (57) into a second-quantization form, where macroscopic quantization has been applied to the electromagnetic field and microscopic quantization to the guest atoms.

First start with atomic Hamiltonian Eq. (56). Following (standard) quantum mechanics, electron coordinates $\mathbf{r}_{m j}(t)$ and their canonical momenta $\mathbf{p}_{m j}(t)$ [Eq. (47)], as well as polarization field $\mathbf{P}_{\mathrm{g}}$, become operators that work on the atomic wave functions. The single-atom wave functions can be expanded in terms of eigenfunctions (labeled $k$ ) of the single-atom Hamiltonian:

$$
\Psi_{m}\left(\mathbf{r}_{1}, \mathbf{r}_{2}, \ldots, \mathbf{r}_{Z_{m}} ; t\right)=\sum_{k} c_{m k}(t) \psi_{m k}\left(\mathbf{r}_{1}, \mathbf{r}_{2}, \ldots, \mathbf{r}_{Z_{m}}\right) .
$$

Second-quantization notation can now be introduced by promoting probability amplitudes $c_{m k}(t)$ and $c_{m k}^{*}(t)$ to become annihilation and creation operators with standard anticommutation relations. These operators become the atomic canonical variables in the second-quantization picture. (One could even go back and start with a Lagrangian that identifies $\Psi_{m}$ and $\Psi_{m}^{*}$ as canonical conjugates [22].) The atomic operators can be written as sums over matrix elements. For example, the atomic Hamiltonian of atom $m$ in standard second-quantization notation is $H_{\mathrm{at}}^{(m)}=\Sigma_{k} E_{k}^{(m)} c_{m k}^{\dagger} c_{m k}$.

The vector potential was again chosen generalized transverse and its canonically conjugate field (46) turned out to be transverse again so that these field operators can be expanded in terms of generalized transverse modes, as in Eqs. (19a) and (19b). The creation and annihilation operators are written as $a_{\lambda}^{\dagger}$ and $a_{\lambda}$, respectively, now that atoms are present. Radiative part (51) of the Hamiltonian becomes $H_{\text {rad }}$ $=\Sigma_{\lambda} \hbar \omega_{\lambda}\left(a_{\lambda}^{\dagger} a_{\lambda}+\frac{1}{2}\right)$.

Interaction terms $V_{\mathrm{P}}^{(m)}$ and $V_{\mathrm{M}}^{(m)}$ in second-quantization notation become

$$
\begin{aligned}
V_{\mathrm{P}}^{(m)}= & -\int d \mathbf{r} \frac{\mathbf{P}_{\mathrm{g} m}(\mathbf{r}) \cdot \mathbf{D}(\mathbf{r})}{\varepsilon_{0} \varepsilon(\mathbf{r})} \\
= & -i \sum_{\lambda} \sum_{k, k^{\prime}} \sqrt{\frac{\hbar \omega_{\lambda}}{2 \varepsilon_{0}}}\left[a_{\lambda} c_{m k}^{\dagger} c_{m k^{\prime}}\right. \\
& \left.\times \int d \mathbf{r} \mathbf{P}_{\mathrm{g} m, k k^{\prime}}(\mathbf{r}) \cdot \mathbf{f}_{\lambda}(\mathbf{r})-\text { H.c. }\right], \\
V_{\mathrm{M}}^{(m)}= & -\int d \mathbf{r} \mathbf{M}_{\mathrm{g} m}^{\prime}(\mathbf{r}) \cdot \mathbf{B}(\mathbf{r}) \\
= & -\sum_{\lambda} \sum_{k, k^{\prime}} \sqrt{\frac{\hbar}{2 \varepsilon_{0} \omega_{\lambda}}}\left\{a_{\lambda} c_{m k}^{\dagger} c_{m k^{\prime}}\right. \\
& \left.\times \int d \mathbf{r} \mathbf{M}_{\mathrm{g} m, k k^{\prime}}^{\prime}(\mathbf{r}) \cdot\left[\boldsymbol{\nabla} \times \mathbf{f}_{\lambda}(\mathbf{r})\right]+\text { H.c. }\right\} .
\end{aligned}
$$

Quantity $\mathbf{P}_{\mathrm{g} m, k k^{\prime}}(\mathbf{r})$ in Eq. (59a) is the matrix element of polarization field $\mathbf{P}_{\mathrm{g} m}(\mathbf{r})$ with respect to states $\psi_{m k}$ and $\psi_{m k^{\prime}}$ of atom $m$. The polarization field couples to field $-\mathbf{D} /\left[\varepsilon_{0} \varepsilon(\mathbf{r})\right]$, which is unequal to $-\mathbf{E} / \varepsilon_{0}$. The interpretation of the interaction is subtle since in definition (42) of the displacement field the polarization of the guest atoms is included. Interaction $V_{\mathrm{P}}^{(m)}$ therefore includes a self-interaction of the polarization field. However, in the expansion in Eq. (59a) of the interaction in terms of the optical modes, dielectric function $\varepsilon(\mathbf{r})$ drops out and the coupling becomes rather simple.

There are no analogous self-interactions in magnetic interaction $V_{\mathrm{M}}^{(m)}$. The magnetic field and the reduced magnetization field are canonically independent and $\mathbf{M}_{\mathrm{g}}^{\prime}$ is not included in the definition of $\mathbf{B}$. All four terms in the quantum multipolar Hamiltonian (57) have now been given in secondquantization notation.

\section{Dipole approximation}

An atom is much smaller than an optical wavelength; its spatial structure cannot be probed with light. One can make 
the well-known assumption that the polarization and magnetization fields associated with the atom are concentrated in its center of mass $\mathbf{R}_{m}$ (the nucleus, say). Mathematically, this means that the integrands in Eqs. (41a) and (41b) are approximated by their values in $u=0$. These two values are the first terms of two infinite Taylor expansions in terms of variable $u$. The dipole approximation is made by keeping only the first term.

Incidentally, the next terms in the Taylor expansions would describe quadrupole interactions, which can be important when the guest atoms are not real atoms but other (larger) quantum systems in interaction with the electromagnetic field. For example, quantum dots (artificial atoms) are much larger than real atoms and so their dipole moments can be much larger as well [42]. Quadrupole moments are more important for quantum dots than for real atoms, especially when excited in their near field by a scanning near-field optical microscope [43]. In the following, however, quadrupole and higher-order moments are neglected.

In the dipole approximation, the magnetization (reduced or not) becomes identically zero and the polarization field becomes

$$
\mathbf{P}_{\mathrm{g} m}(\mathbf{r})=\delta\left(\mathbf{r}-\mathbf{R}_{m}\right) \sum_{k, k^{\prime}} c_{m k}^{\dagger} \boldsymbol{\mu}_{n k^{\prime}}^{(m)} c_{m k^{\prime}},
$$

where the atomic dipole matrix elements $\boldsymbol{\mu}_{k k^{\prime}}^{(m)}$ of guest atom $m$ are defined as

$$
\boldsymbol{\mu}_{k k^{\prime}}^{(m)} \equiv-e\left\langle\psi_{m k}\left|\sum_{j}\left(\mathbf{r}_{m j}-\mathbf{R}_{m}\right)\right| \psi_{m k^{\prime}}\right\rangle .
$$

With Eq. (59a), it follows that in the dipole approximation the interaction energy of an atom with the electromagnetic field in an inhomogeneous dielectric equals

$$
\begin{aligned}
V_{\mathrm{dip}}^{(m)} & =-\sum_{k k^{\prime}} c_{m k}^{\dagger} c_{m k^{\prime}} \boldsymbol{\mu}_{k k^{\prime}}^{(m)} \cdot \mathbf{D}\left(\mathbf{R}_{m}\right) /\left[\varepsilon_{0} \varepsilon\left(\mathbf{R}_{m}\right)\right] \\
& =-i \sum_{\lambda} \sum_{k k^{\prime}} \sqrt{\frac{\hbar \omega_{\lambda}}{2 \varepsilon_{0}}}\left[a_{\lambda} c_{m k}^{\dagger} c_{m k^{\prime}} \boldsymbol{\mu}_{k k^{\prime}}^{(m)} \cdot \mathbf{f}_{\lambda}\left(\mathbf{R}_{m}\right)-\text { H.c. }\right] .
\end{aligned}
$$

This gives the important result that inside an inhomogeneous dielectric, a dipole couples to field $-\mathbf{D} /\left[\varepsilon_{0} \varepsilon(\mathbf{r})\right]$. This generalization of the free-space dipole coupling $[13,22,23]$ was also found in Refs. $[17,18]$. As we shall see in the following section V E, local-field effects can have a strong influence on this interaction. Still, local-field effects are often neglected in macroscopic quantization theories [15,17-19].

\section{E. In need of a local-field model}

In Sec. V C it was not stressed that atomic Hamiltonian (56), in general, is different for an atom in a dielectric and in free space. The potential energy in Eq. (56), which includes the Coulomb potential, is reduced by a factor $\varepsilon(\mathbf{r})$ as compared to free space. (Such a reduction factor is well known for dielectric-filled capacitors.) As a consequence, energy levels and wave functions will be different in a dielectric. This point is missed if one starts with a second-quantized description, for example, when introducing in the medium a "two-level atom" with known transition frequency and dipole moment.

First suppose that the dielectric function is a macroscopically averaged quantity that does not change on atomic length scales. Then, $\varepsilon(\mathbf{r})$ must be unchanged by introducing a guest atom and Hamiltonian (56) can be approximated by

$$
H_{\mathrm{at}}^{(m)} \approx \frac{1}{2 m_{\mathrm{e}}} \sum_{j} p_{m j}^{2}+\frac{1}{2 \varepsilon_{0} \varepsilon\left(\mathbf{R}_{m}\right)} \int d \mathbf{r} P_{\mathrm{g} m}^{2} .
$$

If the guest atom were a hydrogen atom then its Bohr radius and dipole moments would increase by a factor $\varepsilon\left(\mathbf{R}_{m}\right)$ and its energy levels would be reduced by the same factor, according to this Hamiltonian. Any visible line in free space would then be shifted to the infrared in a dielectric. The consequences of approximation (63) would be that the dielectric has a huge effect on the atom's electronic properties. Now the reduction of the Coulomb potential (as well as its screening as a function of distance) is a well-studied subject in solid-state physics [44]. Sometimes one finds the full reduction [as described by Hamiltonian (63)] while in other cases no reduction is found at all.

In general, outer electronic states of atoms will be more affected by the dielectric than the core electrons. An important reason for this is the dispersive interaction of the guest atoms with the atoms that make up the medium. However, such frequency dispersion in the medium is neglected in the present formalism. We should therefore not have the ambition to find an atomic Hamiltonian that leads to correct inner and outer electronic states, including medium effects. A modest model is needed that meets the requirement that energy levels taking part in the optical transitions under study should come out right. Such a model might be obtained by assuming that the atom sits inside an atom-sized cavity with a relative dielectric function $\varepsilon\left(\mathbf{R}_{m}\right)$ that is constant inside the cavity; in general, $\varepsilon\left(\mathbf{R}_{m}\right)$ will be different both from the macroscopic dielectric function just outside the cavity, and unity (the free-space value). More ambitious descriptions of medium effects on atomic Hamiltonians require at least that dispersion of the dielectric is taken into account, perhaps starting from a microscopic model of the dielectric [33-35].

A well-known case, where reduction of potential energy is important, occurs when doping solid silicon with phosphorus to make an $n$-type semiconductor. The high dielectric constant of $\mathrm{Si}(\varepsilon=11.7)$ reduces the potential energy between the outermost electron and the rest of the P-atom, so that the electron can enter the conduction band relatively easily, leaving a $P^{+}$ion [45]. As said before, modifications other than (63) of the atomic Hamiltonian are possible. An important example of the other extreme case, where a reduction of the Coulomb interaction is absent, will be given shortly.

We are interested in atomic lifetime changes and line shifts caused by the medium. In general, the medium induces changes both in atomic Hamiltonian (56) and in the atomfield interactions, as compared to free space. Effects of the medium, which are caused by changes in the atomic Hamil- 
tonian will be called electronic effects. Changes in atomic dipole moments are an example of electronic effects. On the other hand, effects due to modified interactions $V_{\mathrm{P}}^{(m)}$ and $V_{\mathrm{M}}^{(m)}$ between field and atom will be called photonic effects. It is the photonic effects, the changes due to altered properties of the electromagnetic field, which are of primary interest here and in photonics at large. However, only if the electronic changes of the atoms are somehow either absent or accounted for, can one study the photonic effects. In this respect, it is fortunate that line shifts due to changes in the interactions (radiative or Lamb shifts) in a medium usually are too small to be observable and electronic line shifts dominate. Line shifts can therefore be used to estimate medium-induced changes in the atomic Hamiltonian. Given a line shift, one could assign an effective dielectric function $\varepsilon\left(\mathbf{R}_{m}\right)$ for the atomic cavity that produces the observed transition frequency when inserted in the atomic Hamiltonian Eq. (63).

The distinction between photonic and electronic effects is also very important in the interpretation of experiments. For example, the recently observed fivefold reduction of spontaneous-emission rates inside photonic crystals [46] is a photonic effect since possible changes in dipole moments were divided out by choosing a reference sample with identical electronic effects [47]. Some earlier observations of long lifetimes in photonic crystals must be attributed to electronic effects [48].

When studying photonic effects of a medium, the guest atoms ideally are electronically the same as in free space, in particular, with the same eigenfrequencies and transition dipole moments. In that ideal case, which we refer as the empty-cavity model, we have

$$
\varepsilon\left(\mathbf{R}_{m}\right)=1 \quad \forall m .
$$

The atomic Hamiltonian is as in approximation Eq. (63), now with $\varepsilon\left(\mathbf{R}_{m}\right)$ equal to 1 . In other words, guest atoms can only be ideal if the dielectric function is locally changed to the free-space value 1 . The atom, as in free space, sits inside an empty cavity inside the dielectric. A reduction of the intraatomic Coulomb interaction is completely absent in this empty-cavity model. The formation of such a cavity is beyond the scope of the present macroscopic theory. This would require microscopic theories of the dielectric, involving the Pauli exclusion principle for electrons of both the dielectric and the guest atoms.

The empty-cavity model captures the observed absence of large electronic effects of the dielectric on atomic properties of interest but at the same time, the model has consequences for photonic properties: the local changes in $\varepsilon$ will give local changes in mode functions $\mathbf{f}_{\lambda}$ and therefore in dipole coupling Eq. (62). Atomic spontaneous-emission rates will get local-field corrections. These predictions can be tested experimentally. An important example is the emission rate of an atom inside an atomic-sized empty cavity in an otherwise homogeneous medium. To be precise, $\varepsilon(\mathbf{R})=\varepsilon$ for $\mathbf{R}$ not coinciding with any of the $\mathbf{R}_{m}$. The emission rate is $[3 \varepsilon /(2 \varepsilon+1)]^{2} \sqrt{\varepsilon} \Gamma_{0}$, where $\Gamma_{0}$ is the free-space emission rate [16]. The well-known in-medium enhancement by a fac- tor $\sqrt{\varepsilon}$ is further enhanced by the square of a so-called localfield factor. Here, the term between the square brackets is the empty-cavity local-field factor.

In a recent study [49] of refractive-index dependent spontaneous-emission rates, atoms were embedded in a lowindex molecular complex so as to electronically separate them from the medium. For the interpretation of the results, it was important that atomic spectra and dipole moments did not change appreciably while varying the refractive index. The empty-cavity local-field factor was indeed observed [49]. This result is a justification for the macroscopic quantization theory for nondispersive dielectrics.

For inhomogeneous dielectrics, it is in general not easy to calculate local-field factors, either in the empty-cavity model (64) or in other models. The simplest assumption in the empty-cavity model is that the position-dependent local-field factors will have values $3 \varepsilon_{\mathrm{b}}\left(\mathbf{R}_{m}\right) /\left[2 \varepsilon_{\mathrm{b}}\left(\mathbf{R}_{m}\right)+1\right]$, where $\varepsilon_{\mathrm{b}}\left(\mathbf{R}_{m}\right)$ is the bulk dielectric function around atom $m$. The assumption will probably break down when $\varepsilon_{\mathrm{b}}(\mathbf{R})$ varies strongly on the scale of the wavelength of light.

The atomic Hamiltonian can be changed in many ways and consequently, empty-cavity factors are not the only local-field factors that can be obtained from the present macroscopic quantization formalism. One could give up the macroscopic quantization as being too phenomenological and instead, describe the microscopic constituents of the dielectric in the vicinity of the guest atom. This could lead to other local-field factors, depending on the question whether the guest atom sits inside a real cavity inside the dielectric [of which empty cavity (64) is a special case] or not. For homogeneous dielectrics, see Refs. [33,50,51] and references therein. However, for inhomogeneous dielectrics it will be hard to tie a local microscopic approach to the macroscopic description of the inhomogeneous medium on a larger scale.

\section{DIPOLE-COUPLING CONTROVERSY}

Many papers appeared in the 1980s about the equivalence of the minimal-coupling and the multipolar Hamiltonian in free space, for example Refs. [20-24]. The Hamiltonians sometimes lead to different results in calculations. Some authors argued that the minimal-coupling Hamiltonian was to be preferred, while others proposed to refrain from using gauge-dependent equations to stop the confusion. In the multipolar picture, a controversy arose whether a dipole in free space couples to minus of the displacement field, $-\boldsymbol{\mu} \cdot \mathbf{D} / \varepsilon_{0}$, or to the transverse part of the electric field, $-\boldsymbol{\mu} \cdot \mathbf{E}^{\mathrm{T}}$. The first answer is correct and the book by CohenTannoudji et al. helped to settle the argument [13]. It may be useful to give two sources of confusion even for an atom in free space and to compare the free-space dipole coupling with its in-medium generalization Eq. (62).

The main source of confusion is related to approximations. It was found in Sec. V D that a dipole couples to minus of the displacement field:

$$
-\mathbf{D}\left(\mathbf{R}_{m}\right) /\left[\varepsilon_{0} \varepsilon\left(\mathbf{R}_{m}\right)\right]=-\frac{\left[\varepsilon\left(\mathbf{R}_{m}\right) \mathbf{E}\left(\mathbf{R}_{m}\right)+\mathbf{P}_{\mathrm{g}}\left(\mathbf{R}_{m}\right)\right]}{\varepsilon_{0} \varepsilon\left(\mathbf{R}_{m}\right)},
$$


where $\mathbf{P}_{\mathrm{g}}$ is the polarization field of the guest atom itself. In free space, or when assuming an empty-cavity model, a dipole couples to field $-\mathbf{D} / \varepsilon_{0}$. Still, one can find references stating that in free space or in a dielectric [16], a dipole couples to minus of the electric field. The origin of this mistaken interpretation can be traced back to the normal-mode expansion of the displacement field operator for the dielectric with guest atoms

$$
\mathbf{D}(\mathbf{r})=i \varepsilon_{0} \varepsilon(\mathbf{r}) \sum_{\lambda} \sqrt{\frac{\hbar \omega_{\lambda}}{2 \varepsilon_{0}}}\left[a_{\lambda} \mathbf{f}_{\lambda}(\mathbf{r})-a_{\lambda}^{\dagger} \mathbf{f}_{\lambda}^{*}(\mathbf{r})\right] .
$$

The expanded form of the displacement operator is almost the same as in a dielectric without guest atoms [minus Eq. (19b)] but the two differences will now be discussed. The first difference, which also plays a role for free space, is that the equations of motion of the creation and annihilation operators in Eq. (66) have terms involving the atomic variables that the equations of motion of their counterparts $a_{\lambda}^{(0)}$ and $a_{\lambda}^{(0) \dagger}$ for the field without guests do not have. If one approximates displacement field (66) by replacing all the $a_{\lambda}$ and $a_{\lambda}^{\dagger}$ by $a_{\lambda}^{(0)}$ and $a_{\lambda}^{(0) \dagger}$, respectively, then in free space the displacement field (66) is equal to the electric field in the absence of the guest atoms. In other words, if the guest atoms are taken into account in Maxwell's equations, then one finds a dipole coupling to the displacement field, whereas a coupling to the electric field is found when guest atoms are left out of Maxwell's equations. It depends on the observable under study whether the difference between the two dipole couplings can be neglected or not.

The second difference between displacement field (66) (with guests) and its counterpart (no guests) is that mode functions will be changed locally when guest atoms are present, as discussed previously. This difference does not show up in the free-space discussion, of course. In a dielectric, the above approximation of replacing the creation and annihilation operators does not make the displacement field equal to the electric field. The replacement would only have this effect for positions $\mathbf{r}$ in the medium where $\varepsilon(\mathbf{r})$ equals 1 and where local-field effects can be neglected.

Apart from the main source of confusion, there is another reason why the interpretation of the dipole coupling can be confusing: there are two essentially different procedures to go from a minimal-coupling Hamiltonian to a dipole Hamiltonian. The first procedure is to rewrite a minimal-coupling Hamiltonian as a multipolar or dipole Hamiltonian by canonical transformations of its variables (different variables, same Hamiltonian and states). The second procedure is a unitary change of picture (different Hamiltonian, different states, same expectation values). Unlike canonical transformations, picture changes have no classical analogues. Confusion is likely to arise when after a canonical change, a Hamiltonian has exactly the same form as after a picture change. The differences between the two procedures are excellently presented in Ref. [23] for an atom in free space. Either of the two procedures could be chosen for dielectrics as well; in this paper, the PZW transformation of the Lagrangian was used instead.

\section{INHOMOGENEOUS MAGNETIC MEDIA}

The present formalism can be generalized to dielectrics with inhomogeneous magnetic properties as well, where the magnetic permeability becomes $\mu_{0} \mu(\mathbf{r})$ rather than $\mu_{0}$. (So, here " $\mu$ " does not represent the magnitude of a dipole $\boldsymbol{\mu}$.) Such generalizations are even more interesting now that socalled left-handed materials [52] have become the subject of intense scientific discussions, after a prediction that a perfect lens could be made with them [53]. Both $\varepsilon(\mathbf{r})$ and $\mu(\mathbf{r})$ of left-handed materials are negative. Index of refraction $n(\mathbf{r})$ is also negative and this leads to many peculiar properties. Left-handed materials will influence spontaneous-emission rates of nearby guest atoms in different ways than their righthanded counterparts [54].

Generalizations to magnetic media were already considered in Refs. [17,18] and it is relatively straightforward to incorporate position-dependent permeabilities in the formalism of this paper, as we will see now. When $\mu$ becomes position dependent, then the only term that will change in the classical multipolar Hamiltonian (57) is magnetic-field energy $\int d \mathbf{r B}^{2}(\mathbf{r}) /\left[2 \mu_{0} \mu(\mathbf{r})\right]$. The quantum-mechanical description can again be carried out by choosing the generalized Coulomb gauge for the vector potential. Without guest atoms, the vector potential satisfies the source-free wave equation

$$
\boldsymbol{\nabla} \times\left[\frac{1}{\mu(\mathbf{r})} \boldsymbol{\nabla} \times \mathbf{A}(\mathbf{r})\right]+\frac{\varepsilon(\mathbf{r})}{c^{2}} \ddot{\mathbf{A}}(\mathbf{r})=0 .
$$

The electromagnetic field can be expanded in terms of true modes $\mathbf{m}_{\nu}$ different from modes $\mathbf{f}_{\lambda}$. These modes are the harmonic solutions of wave equation (67) and so, they are generalized transverse, just like modes $\mathbf{f}_{\lambda}$ of the nonmagnetic medium. Canonical fields can be found by calculating constrained functional derivatives in the same way as presented in Sec. IV A. It is this point that makes the generalization to magnetic media relatively simple. In second-quantization notation, the electromagnetic field energy becomes $\Sigma_{\nu} \hbar \omega_{\nu}\left(d_{\nu}^{\dagger} d_{\nu}+\frac{1}{2}\right)$, where $d_{\nu}^{\dagger}$ is the creation operator of a photon in mode $\mathbf{m}_{\nu}(\mathbf{r})$. These modes can have mode profiles that differ much from any of modes $\mathbf{f}_{\lambda}(\mathbf{r})$, but otherwise the theoretical description of the medium is not much different. In electric and magnetic interactions (59a) and (59b) of the electromagnetic field with guest atoms, the modes $\mathbf{f}_{\lambda}$ can just be replaced by $\mathbf{m}_{\nu}$ and operators $c_{\lambda}^{(\dagger)}$ by $d_{\nu}^{(\dagger)}$ in order to take both the electric and magnetic properties of the medium into account. As for nonmagnetic media, in the dipole approximation magnetic interaction (59b) is zero; electric dipole interaction (59a) dominates, except for optical transitions with zero dipole moments. When dipole moments are nonzero, the main effect of the dielectric becoming magnetic comes from the change in the mode functions.

\section{SUMMARY AND DISCUSSION}

The aim of the paper was to find a Hamiltonian of guest atoms in an inhomogeneous dielectric, with a multipolar in- 
teraction between the atoms and the electromagnetic field. The multipolar Hamiltonian is simpler than the minimalcoupling Hamiltonian because in the former all interactions between the atoms are mediated by the retarded electromagnetic field. The main results are therefore the quantum multipolar interaction Hamiltonian (57) together with its dipole approximation (62). With this Hamiltonian, one can study how an inhomogeneous dielectric environment can change quantum optical processes of resonant atoms.

In order to obtain the central results, first the electromagnetic field was quantized in the absence of guest atoms. This has certainly been carried out before $[15-17,36]$ but after explaining why real optical mode functions can be used whenever $\varepsilon(\mathbf{r})$ is real, the quantization becomes simpler than found in Refs. [16,17,36]. In particular, only when real mode functions are chosen are the associated generalized positions and momenta Hermitian [see Eq. (16)].

Guest atoms were introduced into the theory such that Maxwell's equations hold with the atomic charge and current densities as source terms. A minimal-coupling Lagrangian that gives rise to these equations was easily written down. However, after choosing a generalized Coulomb gauge, it was not directly clear how to obtain all Maxwell's equations in this particular gauge. In Sec. IV, it was explained that the usual functional derivative must be replaced by a "constrained functional derivative" after choosing a gauge. This is for mathematical reasons rather than a matter of taste or convenience. Simple rules are given to actually compute these constrained functional derivatives. As a result, the gauge-independent Maxwell's equations were found to indeed hold in the generalized Coulomb gauge as well [see Eq. (39)].

There is a second advantage of our careful treatment of functional differentiation. In the multipolar formalism, the field in the dielectric canonically conjugate to the vector potential could relatively easily and unambiguously be identified as minus of the full displacement field [see Eq. (46)]. This field contains the polarization fields produced by both the dielectric and the guest atoms.

Another important result is that the macroscopic description of the dielectric could only be tied up to the microscopic description of the atoms by assuming that the dielectric function is locally modified by the presence of the guest atoms. In particular, in the empty-cavity model [Eq. (64)], the dielectric function has value 1 where wave functions of the guest atoms are nonzero. The local modification of the di- electric function will also change the dipole coupling, giving rise to local-field effects in spontaneous-emission rates. If one would start with a two- or three-level description for the guest atoms, then one implicitly already assumes a local-field model for the dielectric function. Consistency requires one to also choose that local-field model when calculating the mode functions in the dipole interaction (62).

The application of the Power-Zienau-Woolley transformation to the minimal-coupling Lagrangian was shown to produce the multipolar Lagrangian, after the generalized Coulomb gauge had been chosen. Actually, the gauge was chosen earlier than strictly necessary: the choice could have been postponed until the canonical momenta were determined from the multipolar Lagrangian. The story would have been simpler up to that point. The difficulty to find all Maxwell's equations would then show up only after obtaining the multipolar Lagrangian. The reason to first choose the gauge and then do the PZW transformation is that it shows more clearly that the difficulty to find all Maxwell's equations was a consequence of choosing the gauge rather than a consequence of the PZW transformation.

More generally, the presentation given here is one among many possibilities. When going from a classical minimalcoupling Lagrangian to a quantum mechanical multipolar Hamiltonian, one has to make four steps: one step is to choose a gauge, another step is to transform the theory to the multipolar formalism. Yet another step is made when going from a Lagrangian to a Hamiltonian and quantization and second quantization together are step number four. These are now given in the order in which they occurred in this paper but the steps can be interchanged. Not all of the 24 permutations are convenient, but all routes should lead to the equivalent final results. It was shown in detail in Ref. [21] that step two and three can be interchanged for free space: the PZW transformation of the minimal-coupling Lagrangian is equivalent to a picture change of the minimal-coupling Hamiltonian. The equivalence will also hold for inhomogeneous dielectrics.

\section{ACKNOWLEDGMENTS}

We would like to thank Allard Mosk, Rudolf Sprik, and Willem Vos for stimulating discussions. This work was part of the research program of the Stichting voor Fundamenteel Onderzoek der Materie, which was financially supported by the Nederlandse Organisatie voor Wetenschappelijk Onderzoek.
[1] E.M. Purcell, Phys. Rev. 69, 681 (1946).

[2] K.H. Drexhage, J. Lumin. 1-2, 693 (1970).

[3] P.W. Milonni, The Quantum Vacuum (Academic Press, San Diego, 1994), p. 183.

[4] D. Kleppner, Phys. Rev. Lett. 47, 233 (1981).

[5] E. Yablonovitch, Phys. Rev. Lett. 58, 2059 (1987); S. John, ibid. 58, 2486 (1987).

[6] G. Kurizki, A.G. Kofman, and V. Yudson, Phys. Rev. A 53, R35 (1996).

[7] P. Andrew and W.L. Barnes, Science 290, 785 (2000).
[8] G. Kurizki, Phys. Rev. A 42, 2915 (1990).

[9] S. John and J. Wang, Phys. Rev. B 43, 12772 (1991).

[10] D.K. Mynbaev and L.L. Scheiner, Fiber-Optic Communications (Prentice-Hall, Upper Saddle River, NJ, 2001), p. 63.

[11] R. Loudon, The Quantum Theory of Light (Clarendon Press, Oxford, 1983).

[12] D.P. Craig and T. Thirunamachandran, Molecular Quantum Electrodynamics (Academic Press, London, 1984).

[13] C. Cohen-Tannoudji, J. Dupont-Roc, and G. Grynberg, Photons and Atoms. Introduction to Quantum Electrodynamics 
(Wiley, New York, 1989).

[14] L. Mandel and E. Wolf, Optical Coherence and Quantum Optics (Cambridge University Press, Cambridge, 1995).

[15] L. Knöll, W. Vogel, and D.-G. Welsch, Phys. Rev. A 36, 3803 (1987).

[16] R.J. Glauber and M. Lewenstein, Phys. Rev. A 43, 467 (1991).

[17] B.J. Dalton, E.S. Guerra, and P.L. Knight, Phys. Rev. A 54, 2292 (1996).

[18] B.J. Dalton and M. Babiker, Phys. Rev. A 56, 905 (1997).

[19] G. Kweon and N.M. Lawandy, Opt. Commun. 118, 388 (1995).

[20] E.A. Power and T. Thirunamachandran, Am. J. Phys. 46, 370 (1978).

[21] M. Babiker and R. Loudon, Proc. R. Soc. London, Ser. A 385, 439 (1983).

[22] E.A. Power and T. Thirunamachandran, Phys. Rev. A 28, 2649 (1983).

[23] J.R. Ackerhalt and P.W. Milonni, J. Opt. Soc. Am. B 1, 116 (1984).

[24] E.A. Power and T. Thirunamachandran, J. Opt. Soc. Am. 2, 1100 (1985).

[25] S.M. Barnett and R. Loudon, Quantum Semiclassic. Opt. 10, 591 (1998).

[26] N. Vats, S. John, and K. Busch, Phys. Rev. A 65, 043808 (2002).

[27] B. Huttner and S.M. Barnett, Phys. Rev. A 46, 4306 (1992).

[28] M. Wubs and L.G. Suttorp, Phys. Rev. A 63, 043809 (2001).

[29] H.T. Dung, L. Knöll, and D.-G. Welsch, Phys. Rev. A 57, 3931 (1998).

[30] S. Scheel, L. Knöll, and D.-G. Welsch, Phys. Rev. A 58, 700 (1998).

[31] S. Savasta, O. Di Stefano, and R. Girlanda, Phys. Rev. A 65, 043801 (2002).

[32] H.T. Dung, L. Knöll, and D.-G. Welsch, Phys. Rev. A 65, 043813 (2002).

[33] J. Knoester and S. Mukamel, Phys. Rev. A 40, 7065 (1989).
[34] S.-T. Ho and P. Kumar, J. Opt. Soc. Am. B 10, 1620 (1993).

[35] G. Juzeliūnas, Phys. Rev. A 53, 3543 (1996).

[36] W. Vogel and D.-G. Welsch, Lectures on Quantum Optics (Akademie Verlag, Berlin, 1994).

[37] A. Lagendijk and B.A. van Tiggelen, Phys. Rep. 270, 143 (1996).

[38] C.L.A. Hooijer, PhD thesis, Free University, 2001.

[39] J.D. Jackson, Classical Electrodynamics (Wiley, New York, 1975).

[40] C. Itzykson and J.-B. Zuber, Quantum Field Theory (McGrawHill, New York, 1980), p. 456.

[41] P.M. Morse and H. Feschbach, Methods of Theoretical Physics (McGraw-Hill, New York, 1953), Pt. I, p. 53.

[42] J.R. Guest, T.H. Stievater, X. Li, J. Cheng, D.G. Steel, D. Gammon, D.S. Katzer, D. Park, C. Ell, A. Thränhardt, G. Khitrova, and H.M. Gibbs, Phys. Rev. B 65, 241310 (2002).

[43] J.R. Zurita-Sánchez and L. Novotny, J. Opt. Soc. Am. B 19, 1355 (2002).

[44] W. Jones and N.H. March, Theoretical Solid State Physics (Dover, New York, 1985), p. 985.

[45] M.P. Marder, Condensed Matter Physics (Wiley, New York, 2000), p. 489.

[46] A.F. Koenderink, L. Bechger, H.P. Schriemer, A. Lagendijk, and W.L. Vos, Phys. Rev. Lett. 88, 143903 (2002).

[47] L. Bechger, A.F. Koenderink, and W.L. Vos, Langmuir 18, 2444 (2002).

[48] Z.-Y. Li and Z.-Q. Zhang, Phys. Rev. B 63, 125106 (2001).

[49] F.J.P. Schuurmans, D.T.N. de Lang, G.H. Wegdam, R. Sprik, and A. Lagendijk, Phys. Rev. Lett. 80, 5077 (1998).

[50] P. de Vries and A. Lagendijk, Phys. Rev. Lett. 81, 1381 (1998).

[51] F.J.P. Schuurmans, P. de Vries, and A. Lagendijk, Phys. Lett. A 264, 472 (2000).

[52] V.G. Veselago, Sov. Phys. Usp. 10, 509 (1968).

[53] J.B. Pendry, Phys. Rev. Lett. 85, 3966 (2000).

[54] V.V. Klimov, Opt. Commun. 211, 183 (2002). 